Vietnam Journal of Mechanics, VAST, Vol.42, No. 1 (2020), pp. 63 - 86

DOI: https://doi.org/10.15625/0866-7136/14701

\title{
FREE VIBRATION OF FG SANDWICH PLATES PARTIALLY SUPPORTED BY ELASTIC FOUNDATION USING A QUASI-3D FINITE ELEMENT FORMULATION
}

\author{
Le Cong Ich $^{1, *}$, Pham Vu Nam ${ }^{2,3}$, Nguyen Dinh Kien ${ }^{3,4}$ \\ ${ }^{1}$ Le Quy Don Technical University, Hanoi, Vietnam \\ ${ }^{2}$ Thuyloi University, Hanoi, Vietnam \\ ${ }^{3}$ Graduate University of Science and Technology, VAST, Hanoi, Vietnam \\ ${ }^{4}$ Institute of Mechanics, VAST, Hanoi, Vietnam \\ *E-mail: ichlecong@gmail.com
}

Received: 19 December 2019 / Published online: 25 March 2020

\begin{abstract}
Free vibration of functionally graded (FG) sandwich plates partially supported by a Pasternak elastic foundation is studied. The plates consist of three layers, namely a pure ceramic hardcore and two functionally graded skin layers. The effective material properties of the skin layers are considered to vary in the plate thickness by a power gradation law, and they are estimated by Mori-Tanaka scheme. The quasi-3D shear deformation theory, which takes the thickness stretching effect into account, is adopted to formulate a finite element formulation for computing vibration characteristics. The accuracy of the derived formulation is confirmed through a comparison study. The numerical result reveals that the foundation supporting area plays an important role on the vibration behavior of the plates, and the effect of the layer thickness ratio on the frequencies is governed by the supporting area. A parametric study is carried out to highlight the effects of material distribution, layer thickness ratio, foundation stiffness and area of the foundation support on the frequencies and mode shapes of the plates. The influence of the side-to-thickness ratio on the frequencies of the plates is also examined and discussed.
\end{abstract}

Keywords: FG sandwich plate, Pasternak foundation, Mori-Tanaka scheme, quasi-3D theory, free vibration, finite element formulation.

\section{INTRODUCTION}

Sandwich structures with high rigidity, low specific weight, excellent vibration characteristics and good fatigue properties have great potential for use in aerospace industry. These structures, usually consist of a core bonded to two skin layers, however encounter the delamination due to the sudden change in the material properties from one layer to another. Thanks to the advanced manufacturing methods [1], functionally graded materials initiated by Japanese scientists in mid-1980 can now be incorporated into sandwich

(C) 2020 Vietnam Academy of Science and Technology 
construction to improve performance of the structures. Functionally graded (FG) sandwich structures can be designed to have a smooth variation of the properties, and this helps to avoid the delaminating problem. Many investigations on the mechanical behavior of FG and FG sandwich plates, the structures considered in this paper, are summarized in the review papers $[2,3]$, the contributions that are most relevant to the present work are briefly discussed below.

Praveen and Reddy [4] took the effect of temperature rise into consideration in their derivation of a first-order shear deformable four-node quadrilateral (Q4) element for nonlinear transient analysis of FG plates. Zenkour [5,6] presented a sinusoidal shear deformation plate theory for bending, buckling and vibration analyses of FG sandwich plates. The effect of the material distribution, side-to-thickness ratio, core thickness on the frequencies are illustrated by the author through a simply supported plate. The theory was then employed by Zenkour and Sobhy [7] to study the thermal buckling of FG sandwich plates with temperature-dependent material properties. A n-order shear deformation theory was proposed by Xiang et al. [8] for free vibration analysis of FG sandwich plates. Zero transverse shear stresses at the top and bottom surfaces of plates are satisfied in the theory, and the Reddy's third-order shear deformation theory can be obtained as a special case. The $n$-order shear deformation theory was then used in combination with the meshless global collocation method by Xiang et al. [9] to compute the frequencies of FG sandwich plates. Neves et al. [10] derived a quasi-3D shear deformation theory for analyzing isotropic and FG sandwich plates by taking the extensibility in the thickness direction into account. The collocation with radial basis functions was adopted by the authors to obtain the static and free vibration characteristics of the plates. Various higher-order shear deformation theories for analysis of FG plates were proposed by Thai and his coworkers in [11-13]. In the theories, the transverse displacement is split into two parts, the bending and shear parts. In [14], Thai et al. proposed a new first-order shear deformation theory for analysis of sandwich plates with an isotropic homogeneous core and two FG face layers. The shear stresses in the theory are directly computed from transverse shear forces, and shear correction factors are not necessary to use. Iurlaro et al. [15] adopted the refined zigzag theory to formulate finite element formulations for bending and free vibration analysis of FG sandwich plates. The numerical investigations by the authors showed that the zigzag theory is superior in predicting the mechanical behavior of the plates to the first-order and third-order shear deformation theories. Pandey and Pradyumna [16] employed the higher-order layerwise theory to derive an eight-node isoparametric element for static and dynamic analyses of FG sandwich plates. The numerical results obtained in the work showed the efficiency and accuracy of the derived element in evaluating the bending and dynamic chracteristices of the plates. Belabed et al. [17] proposed a three-unknown hyperbolic shear deformation theory for free vibration study of FG sandwich plates with a homogeneous or FG core. Recently, Daikh and Zenkour [18] considered the effect of porosities in bending behavior of FG sandwich plates. Power law and sigmoid functions are adopted by the authors to describe the variation of the material properties of the FG skin layers.

The effect of elastic foundation support on mechanical behavior of FG and FG sandwich plates has been reported by several authors. In this line of works, Lü et al. [19] 
considered the interaction between plate surface and foundation as the traction boundary conditions of the plate in their free vibration analysis of an FG plate resting on a Pasternak foundation. By expanding the state variables in trigonometric dual series and with the aid of the state space method, the authors obtained an exact solution for a simply supported plate. Also adopting the Pasternak foundation model, Benyoucef et al. [20] studied reponse of a simply supported FG plate on foundation to distributed loads. Equilibrium equations were derived using the hyperbolic shear deformation theory and the Navier solution was employed to obtain the displacements. Various shear deformation theories were employed by Sobhy [21] to study buckling and free vibration of FG sandwich plates resting on a Pasternak foundation. The effects of Winkler and Pasternak foundation parameters on bending of FG plates were considered by Al Khateeb and Zenkour [22], taking the influence of temperature and moisture into account. The influence of tangential edge constraints and foundation support on buckling and postbuckling behaviour of FG sandwich plates and FG sandwich spherical shells was respectively considered by Tung [23], Khoa and Tung [24] using the Galerkin method. Based on a hyperbolic shear and normal deformation plate theory, Akavci [25] carried out static bending, buckling and free vibration analyses of FG sandwich plates supported by a Pasternak foundation. In [26], the effect of neutral surface position was taken into account in studying vibration of a rectangular FG plate resting on an elastic foundation. Bending and vibration analyses of FG plates on an elastic foundation were performed by Benahmed et al. [27] using a quasi-3D hyperbolic shear deformation theory. Free vibration of FG plates on a Pasternak foundation was recently investigated through 2D and quasi-3D shear deformation theories [28].

It has been shown that the frequencies and mode shapes of structures partially supported by an elastic foundation are much different from that of the ones fully supported by the foundation $[29,30]$. The vibration modes of plates, as shown by Motaghian et al. in [31], are governed by the area and position of the foundation support as well. To the authors' best knowledge, the free vibration of FG sandwich plates partially supported by an elastic foundation has not been reported in the literature and it is considered in the present paper. The plates considered herein are composed of three layers, a ceramic core and two FG skin layers. The material properties of the skin layers are assumed to vary in the thickness direction by a simple power gradation law, and they are estimated by Morri-Tanaka scheme. Pasternak foundation model is adopted herein for describing the foundation. Based on a quasi-3D shear deformation theory, a finite element formulation is derived and employed to compute frequencies and mode shapes of the plates. The effects of the material distribution, layer thickness ratio and foundation parameters on the vibration characteristics are investigated in detail. The influence of the side-to-thickness ratio on the frequencies is also examined and discussed.

\section{MATHEMATICAL MODEL}

Fig. 1 shows a rectangular FG sandwich plate with length $a$, width $b$ and thickness $h$, partially supported by an elastic foundation. The Cartersian coordinate system $(x, y, z)$ in the figure is chosen such that the $(x, y)$ plane is coincident with the mid-plane, and $z$-axis directs upward. 


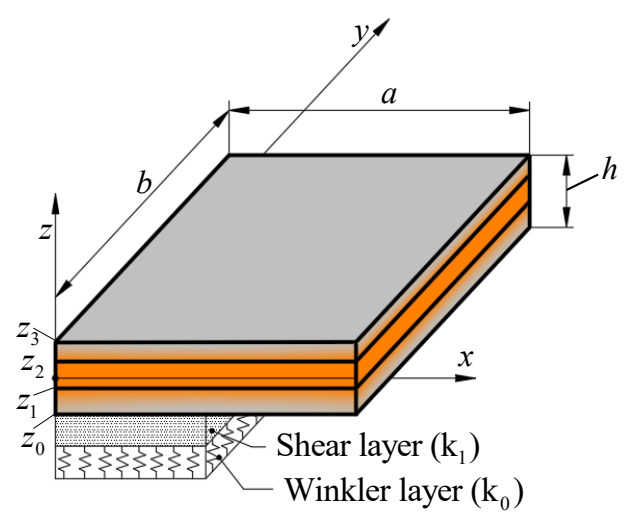

(a)

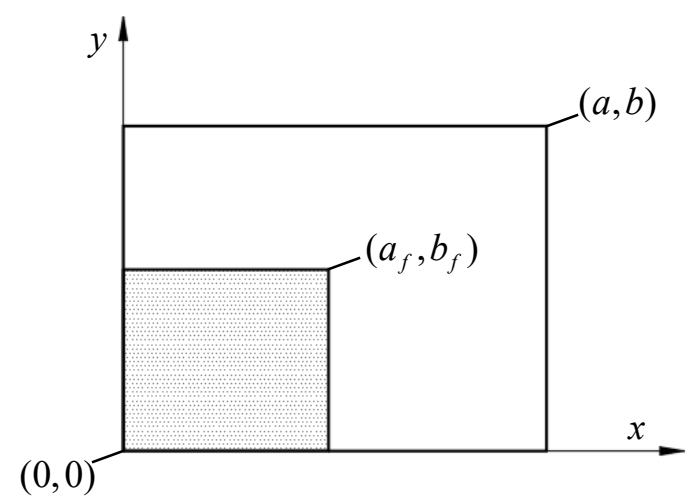

(b)

Fig. 1. FG sandwich plate partially supported by a Pasternak elastic foundation

The plate consists of three layers, a homogeneous ceramic core and two FG metalceramic skin layers. Denoting $z_{0}, z_{1}, z_{2}$ and $z_{3}$ are, respectively, the vertical ordinates of the bottom surface, the two layer interfaces and the top surface, in which $z_{0}=-h / 2$ and $z_{3}=h / 2$. The foundation considered herein is a Pasternak model, which consists of elastic springs with stiffness $k_{0}$ and a shear layer with stiffness $k_{1}$. The foundation area is assumed to be rectangular with length $a_{f}$ and width $b_{f}$, supported the plate at its left corner as shown in Fig. 1(b). The volume fraction of the constituents of the skin layers is supposed to vary in the thickness direction according to

$$
\begin{aligned}
V_{c}^{(1)}(z) & =\left(\frac{z-z_{0}}{z_{1}-z_{0}}\right)^{n} & & , z \in\left[z_{0}, z_{1}\right] \\
V_{c}^{(2)}(z) & =1 & & , z \in\left[z_{1}, z_{2}\right] \\
V_{c}^{(3)}(z) & =\left(\frac{z-z_{3}}{z_{2}-z_{3}}\right)^{n} & & , z \in\left[z_{2}, z_{3}\right] \\
V_{m}^{(k)} & =1-V_{c}^{(k)}, & &
\end{aligned}
$$

where $k=1,2,3 ; V_{m}$ and $V_{c}$ are, respectively, the volume fraction of the metal and ceramic; $n$ is the power-law material index, defining the variation of constituents through the plate thickness. Mori-Tanaka scheme is employed herewith to estimate the effective material properties. According to the Mori-Tanaka scheme, the effective local bulk modulus $K_{f}^{(k)}$ and shear modulus $G_{f}^{(k)}$ of the $k^{\text {th }}$ layer of the sandwich plate can be given by [32]

$$
\frac{K_{f}^{(k)}-K_{m}^{(k)}}{K_{c}^{(k)}-K_{m}^{(k)}}=\frac{V_{c}^{(k)}}{1+\left(1-V_{c}^{(k)}\right)\left(K_{c}^{(k)}-K_{m}^{(k)}\right) /\left(K_{m}^{(k)}+4 G_{m}^{(k)} / 3\right)}
$$




$$
\frac{G_{f}^{(k)}-G_{m}^{(k)}}{G_{c}^{(k)}-G_{m}^{(k)}}=\frac{V_{c}^{(k)}}{1+\left(1-V_{c}^{(k)}\right)\left(G_{c}^{(k)}-G_{m}^{(k)}\right) /\left\{G_{m}^{(k)}+G_{m}^{(k)}\left(9 K_{m}^{(k)}+8 G_{m}^{(k)}\right) /\left[6\left(K_{m}^{(k)}+2 G_{m}^{(k)}\right)\right]\right\}^{\prime}}
$$

where

$$
K_{c}^{(k)}=\frac{E_{c}^{(k)}}{3\left(1-2 \mu_{c}^{(k)}\right)}, G_{c}^{(k)}=\frac{E_{c}^{(k)}}{2\left(1+\mu_{c}^{(k)}\right)}, K_{m}^{(k)}=\frac{E_{m}^{(k)}}{3\left(1-2 \mu_{m}^{(k)}\right)}, G_{m}^{(k)}=\frac{E_{m}^{(k)}}{2\left(1+\mu_{m}^{(k)}\right)} m,
$$

are the local bulk and the shear moduli of the ceramic and metal at the $k^{\text {th }}$ layer, respectively.

Noting that the effective mass density $\rho_{f}^{(k)}$ is defined by Voigt model as

$$
\rho_{f}^{(k)}=\left(\rho_{c}^{(k)}-\rho_{m}^{(k)}\right) V_{c}^{(k)}+\rho_{m}^{(k)}
$$

The effective Young's modulus $E_{f}^{(k)}$ and Poisson's ratio $v_{f}^{(k)}$ are computed via effective bulk modulus and shear modulus as

$$
E_{f}^{(k)}=\frac{9 K_{f}^{(k)} G_{f}^{(k)}}{3 K_{f}^{(k)}+G_{f}^{(k)}}, \quad v_{f}^{(k)}=\frac{3 K_{f}^{(k)}-2 G_{f}^{(k)}}{6 K_{f}^{(k)}+2 G_{f}^{(k)}} .
$$

Based on the quasi-3D shear deformation theory $[12,13]$, the displacements in the $x$-, $y$ - and $z$-directions, $u(x, y, z, t), v(x, y, z, t)$ and $w(x, y, z, t)$, are, respectively, given by

$$
\begin{aligned}
u(x, y, z, t) & =u_{0}(x, y, t)-z w_{b, x}(x, y, t)-f(z) w_{s, x}(x, y, t), \\
v(x, y, z, t) & =v_{0}(x, y, t)-z w_{b, y}(x, y, t)-f(z) w_{s, y}(x, y, t), \\
w(x, y, z, t) & =w_{b}(x, y, t)+w_{s}(x, y, t)+g(z) w_{z}(x, y, t),
\end{aligned}
$$

where $u_{0}(x, y, t)$ and $v_{0}(x, y, t)$ are, respectively, the displacements in $x$ - and $y$-directions of a point on the mid-plane; $w_{b}(x, y, t), w_{s}(x, y, t)$ and $w_{z}(x, y, t)$ are, respectively, bending and shear components of the transverse displacement, and

$$
f(z)=\frac{4}{3 h^{2}} z^{3}, \quad g(z)=1-f_{, z}=1-\frac{4}{h^{2}} z^{2} .
$$

In the above equation and hereafter, a subscript comma is used to denote the derivative with respect to the followed variable, e.g. $f_{z}=\partial f / \partial z$.

The strains resulted from Eq. (7) are of the forms

$$
\begin{aligned}
& \left\{\begin{array}{c}
\varepsilon_{x} \\
\varepsilon_{y} \\
\gamma_{x y}
\end{array}\right\}=\left\{\begin{array}{c}
\varepsilon_{x}^{0} \\
\varepsilon_{y}^{0} \\
\gamma_{x y}^{0}
\end{array}\right\}+z\left\{\begin{array}{c}
\kappa_{x}^{b} \\
\kappa_{y}^{b} \\
\kappa_{x y}^{b}
\end{array}\right\}+f(z)\left\{\begin{array}{c}
\kappa_{x}^{s} \\
\kappa_{y}^{s} \\
\kappa_{x y}^{s}
\end{array}\right\}, \\
& \left\{\begin{array}{c}
\gamma_{x z} \\
\gamma_{y z}
\end{array}\right\}=g(z)\left\{\begin{array}{l}
\kappa_{x z} \\
\kappa_{y z}
\end{array}\right\}, \varepsilon_{z}=g_{, z}(z) w_{z},
\end{aligned}
$$


where

$$
\begin{aligned}
& \varepsilon_{x}^{0}=u_{0, x}, \quad \varepsilon_{y}^{0}=v_{0, y}, \gamma_{x y}^{0}=u_{0, y}+v_{0, x}, \quad \kappa_{x}^{b}=-w_{b, x x}, \quad \kappa_{y}^{b}=-w_{b, y y}, \\
& \kappa_{x y}^{b}=-2 w_{b, x y}, \quad \kappa_{x}^{s}=-w_{s, x x}, \quad \kappa_{y}^{s}=-w_{s, y y}, \quad \kappa_{x y}^{s}=-2 w_{s, x y}, \\
& \kappa_{x z}=\left(w_{s, x}+w_{z, x}\right), \quad \kappa_{y z}=\left(w_{s, y}+w_{z, y}\right) .
\end{aligned}
$$

The constitutive equations based on linear behaviour of the plate material are of the forms

$$
\left\{\begin{array}{c}
\sigma_{x} \\
\sigma_{y} \\
\sigma_{z} \\
\tau_{x y} \\
\tau_{x z} \\
\tau_{y z}
\end{array}\right\}=\left[\begin{array}{cccccc}
Q_{11}^{(k)} & Q_{12}^{(k)} & Q_{12}^{(k)} & 0 & 0 & 0 \\
Q_{12}^{(k)} & Q_{11}^{(k)} & Q_{12}^{(k)} & 0 & 0 & 0 \\
Q_{12}^{(k)} & Q_{12}^{(k)} & Q_{11}^{(k)} & 0 & 0 & 0 \\
0 & 0 & 0 & Q_{44}^{(k)} & 0 & 0 \\
0 & 0 & 0 & 0 & Q_{44}^{(k)} & 0 \\
0 & 0 & 0 & 0 & 0 & Q_{44}^{(k)}
\end{array}\right]\left\{\begin{array}{c}
\varepsilon_{x} \\
\varepsilon_{y} \\
\varepsilon_{z} \\
\gamma_{x y} \\
\gamma_{y z} \\
\gamma_{x z}
\end{array}\right\},
$$

in which

$$
Q_{11}^{(k)}=E_{f}^{(k)} \frac{1-\left(v_{f}^{(k)}\right)^{2}}{1-3\left(v_{f}^{(k)}\right)^{2}-2\left(v_{f}^{(k)}\right)^{3}}, Q_{12}^{(k)}=E_{f}^{(k)} \frac{v_{f}^{(k)}\left(1+v_{f}^{(k)}\right)}{1-3\left(v_{f}^{(k)}\right)^{2}-2\left(v_{f}^{(k)}\right)^{3}}, Q_{44}^{(k)}=G_{f}^{(k)}, G_{f}^{(k)}=\frac{E_{f}^{(k)}}{2\left(1+v_{f}^{(k)}\right)} .
$$

The strain energy stemming from the plate deformation is given by

$$
U^{\mathrm{P}}=\frac{1}{2} \int_{V}\left(\sigma_{x} \varepsilon_{x}+\sigma_{y} \varepsilon_{y}+\sigma_{z} \varepsilon_{z}+\tau_{x y} \gamma_{x y}+\tau_{y z} \gamma_{y z}+\tau_{x z} \gamma_{x z}\right) \mathrm{d} V,
$$

with $V$ is the volume of the plate. Substituting Eqs. (9)-(11) into Eq. (13), one gets

$$
\begin{aligned}
U^{\mathrm{P}}= & \frac{1}{2} \int_{0}^{a} \int_{0}^{b}\left\{A_{11}\left(u_{0, x}^{2}+v_{0, y}^{2}\right)-2 A_{12}\left(u_{0, x} w_{b, x x}+v_{0, y} w_{b, y y}\right)+A_{22}\left(w_{b, x x}^{2}+w_{b, y y}^{2}+\frac{64}{h^{4}} w_{z}^{2}\right)\right. \\
& -\frac{8}{3 h^{2}} A_{23}\left(u_{0, x} w_{s, x x}+v_{0, y} w_{s, y y}\right)+\frac{8}{3 h^{2}} A_{44}\left(w_{b, x x} w_{s, x x}+w_{b, y y} w_{s, y y}\right)+\frac{16}{9 h^{4}} A_{66}\left(w_{s, x x}^{2}+w_{s, y y}^{2}\right) \\
& +2 B_{11} u_{0, x} v_{0, y}-2 B_{12}\left[u_{0, x} w_{b, y y}+v_{0, y} w_{b, x x}+\frac{8}{h^{2}}\left(u_{0, x}+v_{0, y}\right) w_{z}\right]-\frac{8}{3 h^{2}} B_{23}\left(u_{0, x} w_{s, y y}+v_{0, y} w_{s, x x}\right) \\
& +2 B_{22}\left[w_{b, x x} w_{b, y y}+\frac{8}{h^{2}}\left(w_{b, x x}+w_{b, y y}\right) w_{z}\right]-\frac{8}{3 h^{2}} B_{23}\left(u_{0, x} w_{s, y y}+y_{0, y} w_{s, x x}\right) \\
& +B_{44}\left[\frac{8}{3 h^{2}}\left(w_{b, x x} w_{s, y y}+w_{b, y y} w_{s, x x}\right)+\frac{64}{3 h^{4}}\left(w_{s, x x}+w_{s, y y}\right) w_{z}\right]+\frac{32}{9 h^{4}} B_{66} w_{s, x x} w_{s, y y} \\
& +C_{11}\left[\left(u_{0, y}+v_{0, x}\right)^{2}+w_{z, x}^{2}+w_{z, y}^{2}+w_{s, x}^{2}+w_{s, y}^{2}+2\left(w_{s, x} w_{z, x}+w_{s, y} w_{z, y}\right)\right]-4 C_{12}\left(u_{0, y}+v_{0, x}\right) w_{b, x y} \\
& +4 C_{22}\left[w_{b, x y}^{2}-\frac{2}{h^{2}}\left(w_{s, x}^{2}+w_{s, y}^{2}\right)-\frac{4}{h^{2}}\left(w_{s, x} w_{z, x}+w_{s, y} w_{z, y}\right)-\frac{2}{h^{2}}\left(w_{z, x}^{2}+w_{z, y}^{2}\right)\right] \\
& +\frac{16}{h^{4}} C_{44}\left[w_{s, x}^{2}+w_{s, y}^{2}+w_{z, x}^{2}+w_{z, y}^{2}+2\left(w_{s, x} w_{z, x}+w_{s, y} w_{z, y}\right)+\frac{2}{3} h^{2} w_{b, x y} w_{s, x y}\right] \\
& \left.-\frac{16}{3 h^{2}} C_{23}\left(u_{0, y}+v_{0, x}\right) w_{s, x y}+\frac{64}{9 h^{4}} C_{66} w_{s, x y}^{2}\right\} \mathrm{d} x \mathrm{~d} y .
\end{aligned}
$$


In the above equation, $A_{11}, A_{12}, \ldots, C_{44}, C_{66}$ are the plate rigidities, defined as

$$
\begin{gathered}
\left(A_{11}, A_{12}, A_{22}, A_{23}, A_{44}, A_{66}\right)=\sum_{k=1}^{3} \int_{z_{k-1}}^{z_{k}} Q_{11}^{(k)}\left(1, z, z^{2}, z^{3}, z^{4}, z^{6}\right) \mathrm{d} z, \\
\left(B_{11}, B_{12}, B_{22}, B_{23}, B_{44}, B_{66}\right)=\sum_{k=1}^{3} \int_{z_{k-1}}^{z_{k}} Q_{12}^{(k)}\left(1, z, z^{2}, z^{3}, z^{4}, z^{6}\right) \mathrm{d} z, \\
\left(C_{11}, C_{12}, C_{22}, C_{23}, C_{44}, C_{66}\right)=\sum_{k=1}^{3} \int_{z_{k-1}}^{z_{k}} Q_{44}^{(k)}\left(1, z, z^{2}, z^{3}, z^{4}, z^{6}\right) \mathrm{d} z .
\end{gathered}
$$

The strain energy resulted from the foundation deformation is of the form

$$
\begin{aligned}
U^{\mathrm{F}} & =\iint_{S_{F}}\left[k_{0} w_{0}^{2}+k_{1}\left(w_{0, x_{0, x}}^{2}+w_{0, y}^{2}\right)\right] \mathrm{d} S \\
& =\int_{0}^{a_{f}} \int_{0}^{b_{f}}\left\{k_{0}\left(w_{b}+w_{s}+w_{z}\right)^{2}+k_{1}\left[\left(w_{b, x}+w_{s, x}+w_{z, x}\right)^{2}+\left(w_{b, y}+w_{s, y}+w_{z, y}\right)^{2}\right]\right\} \mathrm{d} x \mathrm{~d} y,
\end{aligned}
$$

where $S_{F}$ is the area of the foundation support, and $w_{0}=w(z=0)$.

The total energy $U$ of the plate with the foundation support is

$$
U=U^{\mathrm{P}}+U^{\mathrm{F}} \text {. }
$$

The kinetic energy of the plate resulted from Eq. (7) is of the form

$$
\begin{aligned}
T= & \frac{1}{2} \int_{0}^{a} \int_{0}^{b}\left\{I_{11}\left[\dot{u}_{0}^{2}+\dot{w}_{0}^{2}+\left(\dot{w}_{b}+\dot{w}_{s}\right)^{2}\right]+2 I_{12}\left(\dot{u}_{0} \dot{w}_{b, x}+\dot{w}_{0} \dot{w}_{b, y}\right)\right. \\
& +I_{12}\left[\dot{w}_{b, x}^{2}+\dot{w}_{b, y}^{2}-\frac{8}{h^{2}}\left(\dot{w}_{b}+\dot{w}_{s}+\dot{w}_{z}\right) \dot{w}_{z}\right]-\frac{8}{3 h^{2}} I_{23}\left(\dot{u}_{0} \dot{w}_{s, x}+\dot{v}_{0} \dot{w}_{s, y}\right) \\
& \left.-\frac{8}{3 h^{2}} I_{44}\left(\dot{w}_{b, x} \dot{w}_{s, x}+\dot{w}_{b, y} \dot{w}_{s, y}+\frac{2}{h^{2}} \dot{w}_{z}^{2}\right)+\frac{16}{9 h^{4}}\left(\dot{w}_{s, x}^{2}+\dot{w}_{s, y}^{2}\right)\right\} \mathrm{d} x \mathrm{~d} y,
\end{aligned}
$$

where the mass moments $I_{11}, I_{12}, \ldots, I_{66}$ are defined as

$$
\left(I_{11}, I_{12}, I_{22}, I_{23}, I_{44}, I_{66}\right)=\sum_{k=1}^{3} \int_{z_{k-1}}^{z_{k}} \rho_{f}^{(k)}\left[1, z, z^{2}, z^{3}, z^{4}, z^{6}\right] \mathrm{d} z,
$$

where the effective mass density $\rho_{f}^{(k)}$ is defined by Eq. (5).

Equations of motion for the plate can be obtained by applying Hamilton's principle to Eqs. (17) and (18). However, a closed-form solution for such equations is hardly obtained for the plate partially supported by the elastic foundation. A finite element formulation is derived in the next section for obtaining frequencies and vibration modes of the plate. 


\section{FINITE ELEMENT FORMULATION}

A Q4 plate element with size of $\left(x_{e}, y_{e}\right)$ is derived in this section. In addition to the values of the displacements at the nodes, their derivatives are also taken as degrees of freedom, and the vector of nodal displacements is given by

$$
\underset{(44 \times 1)}{\mathbf{d}}=\left\{\begin{array}{lllll}
\mathbf{d}_{u_{0}} & \mathbf{d}_{v_{0}} & \mathbf{d}_{w_{b}} & \mathbf{d}_{w_{s}} & \mathbf{d}_{w_{z}}
\end{array}\right\}^{T}
$$

where and hereafter, a superscript ' $T$ ' denotes the transpose of a vector or a matrix; $\mathbf{d}_{u_{0}}, \mathbf{d}_{v_{0}}, \mathbf{d}_{w_{b}}, \mathbf{d}_{w_{s}}$ and $\mathbf{d}_{w_{z}}$ are defined as

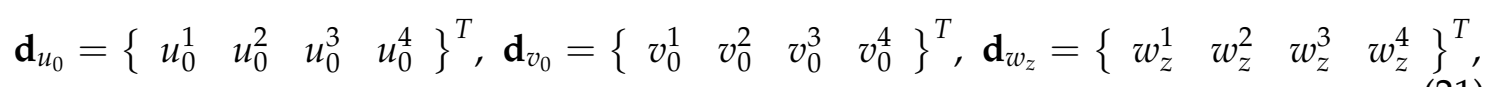

and

$$
\mathbf{d}_{\mathrm{w}_{b}}=\left\{\mathbf{d}_{\mathrm{w}_{b}}^{1} \mathbf{d}_{\mathrm{w}_{b}}^{2} \mathbf{d}_{\mathrm{w}_{b}}^{3} \mathbf{d}_{\mathrm{w}_{b}}^{4}\right\}^{T}, \mathbf{d}_{\mathrm{w}_{s}}=\left\{\mathbf{d}_{\mathrm{w}_{s}}^{1} \mathbf{d}_{\mathrm{w}_{s}}^{2} \mathbf{d}_{\mathrm{w}_{s}}^{3} \mathbf{d}_{\mathrm{ws}^{s}}^{4}\right\}^{T}
$$

with

$$
\mathbf{d}_{\mathrm{w}_{b}}^{j}=\left\{\begin{array}{llll}
w_{b}^{j} & w_{b, x}^{j} & w_{b, y}^{j} & w_{b, x y}^{j}
\end{array}\right\}^{T}, \mathbf{d}_{\mathrm{w}_{s}}^{j}=\left\{\begin{array}{llll}
w_{s}^{j} & w_{s, x}^{j} & w_{s, y}^{j} & w_{s, x y}^{j}
\end{array}\right\}^{T}, j=1, \ldots, 4 .
$$

The stiffness and mass matrices for the element are better to derived in term of the natural coordinates $\xi$ and $\eta: \xi=2\left(x-x_{C}\right) / x_{e}, \eta=2\left(y-y_{C}\right) / y_{e}$, with $\left(x_{C}, y_{C}\right)$ is the centroid coordinates of the element. For $-x_{e} / 2 \leq\left(x-x_{C}\right) \leq x_{e} / 2 \Rightarrow-1 \leq \xi \leq 1$ and $-y_{e} / 2 \leq\left(y-y_{C}\right) \leq y_{e} / 2 \Rightarrow-1 \leq \eta \leq 1$ [33]. In this regard, the relation between the derivatives in the two coordinate systems are given by

$$
\begin{aligned}
& \frac{\partial(.)}{\partial \xi}=\frac{\partial(.)}{\partial x} \frac{\partial x}{\partial \xi}+\frac{\partial(.)}{\partial y} \frac{\partial y}{\partial \xi} \\
& \frac{\partial(.)}{\partial \eta}=\frac{\partial(.)}{\partial x} \frac{\partial x}{\partial \eta}+\frac{\partial(.)}{\partial y} \frac{\partial y}{\partial \eta}
\end{aligned} \text { or }\left\{\begin{array}{l}
(.), \xi \\
(.), \eta
\end{array}\right\}=\mathbf{J}\left\{\begin{array}{l}
(.), x \\
(.)_{, y}
\end{array}\right\} \text { and }\left\{\begin{array}{l}
(.), x \\
(.), y
\end{array}\right\}=\mathbf{J}^{-1}\left\{\begin{array}{l}
(.), \xi \\
(.), \eta
\end{array}\right\},
$$

with the Jacobian matrix $\mathbf{J}$ is of the form

$$
\mathbf{J}=\left[\begin{array}{ll}
x_{, \xi} & y_{, \xi} \\
x_{, \eta} & y_{, \eta}
\end{array}\right]
$$

The displacements $u_{0}, v_{0}$ and $w_{z}$ are interpolated from their nodal values as

$$
u_{0}=\mathbf{N} \mathbf{d}_{u_{0}}=\sum_{i=1}^{4} N_{i} u_{0}^{i}, \quad v_{0}=\mathbf{N d}_{v_{0}}=\sum_{i=1}^{4} N_{i} v_{0}^{i}, \quad w_{z}=\mathbf{N d}_{w_{z}}=\sum_{i=1}^{4} N_{i} w_{z}^{i},
$$

where $N_{i}$ are the Lagrangian functions with the following form

$$
N_{i}=\frac{1}{4}\left(1+\xi_{i} \xi\right)\left(1+\eta_{i} \eta\right) \quad(i=1, \ldots, 4) \quad \text { and } \quad \mathbf{N}=\left[\begin{array}{llll}
N_{1} & N_{2} & N_{3} & N_{4}
\end{array}\right],
$$


As seen from Eqs. (9) and (10), the transverse bending and shear displacements should be twice differentiable, and Hermite polynomials are employed herein to interpolate these displacements as

$$
\begin{aligned}
& w_{b}=\sum_{i=1}^{4} \mathbf{H}_{i} \mathbf{d}_{w_{b}}^{i}=\sum_{i=1}^{4}\left[\begin{array}{llll}
H_{i}^{1} & H_{i}^{2} & H_{i}^{3} & H_{i}^{4}
\end{array}\right]\left\{\begin{array}{llll}
w_{b}^{i} & w_{b, x}^{i} & w_{b, y}^{i} & w_{b, x y}^{i}
\end{array}\right\}^{T},
\end{aligned}
$$

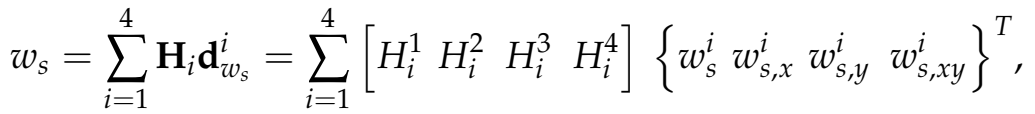

where the interpolation functions $H_{i}^{j}$ have the following forms [34]

$$
\begin{aligned}
H_{i}^{1} & =\frac{1}{16}\left(\xi+\xi_{i}\right)^{2}\left(\xi \xi_{i}-2\right)\left(\eta+\eta_{i}\right)^{2}\left(\eta \eta_{i}-2\right), \\
H_{i}^{2} & =\frac{1}{16} \xi_{i}\left(\xi+\xi_{i}\right)^{2}\left(1-\xi \xi_{i}\right)\left(\eta+\eta_{i}\right)^{2}\left(\eta \eta_{i}-2\right), \\
H_{i}^{3} & =-\frac{1}{16}\left(\xi+\xi_{i}\right)^{2}\left(\xi \xi_{i}-2\right) \eta_{i}\left(\eta+\eta_{i}\right)^{2}\left(\eta \eta_{i}-1\right), \\
H_{i}^{4} & =\frac{1}{16} \xi_{1}\left(\xi+\xi_{i}\right)^{2}\left(\xi \xi_{i}-1\right) \eta_{i}\left(\eta+\eta_{i}\right)^{2}\left(\eta \eta_{i}-1\right), \\
\mathbf{H}_{i} & =\left[\begin{array}{llll}
H_{i}^{1} & H_{i}^{2} & H_{i}^{3} & H_{i}^{4}
\end{array}\right], \quad i=(1, \ldots, 4), \underset{1 \times 16}{\mathbf{H}}=\left[\begin{array}{llll}
\mathbf{H}_{1} & \mathbf{H}_{2} & \mathbf{H}_{3} & \mathbf{H}_{4}
\end{array}\right] .
\end{aligned}
$$

Using the above interpolation scheme, one can write the strain energy $U_{e}$ of the element in terms of the nodal displacement vector $(\mathbf{d})$ as

$$
U_{e}=\frac{1}{2} \sum_{i=1}^{N E_{\mathrm{P}}} \mathbf{d}_{i}^{T} \mathbf{k}_{i}^{\mathrm{P}} \mathbf{d}_{i}+\frac{1}{2} \sum_{i=1}^{N E_{\mathrm{F}}} \mathbf{d}_{i}^{T} \mathbf{k}_{i}^{\mathrm{F}} \mathbf{d}_{i}
$$

where ' $N E_{\mathrm{P}}$ ' and ' $N E_{\mathrm{F}}$ ' are, respectively, the total numbers of elements used to discrete the plate and the foundation; $\mathbf{k}^{\mathrm{P}}$ and $\mathbf{k}^{\mathrm{F}}$ are, respectively, the element stiffness matrices resulted from the plate and the foundation deformation. The stiffness matrix $\mathbf{k}^{\mathrm{P}}$ can be written in sub-matrices as

$$
\mathbf{k}^{\mathrm{P}}=\left[\begin{array}{ccccc}
\mathbf{k}_{u u}^{\mathrm{P}} & \mathbf{k}_{u v}^{\mathrm{P}} & \mathbf{k}_{u w_{b}}^{\mathrm{P}} & \mathbf{k}_{u w_{s}}^{\mathrm{P}} & \mathbf{k}_{u w_{z}}^{\mathrm{P}} \\
\left(\mathbf{k}_{u v}^{\mathrm{P}}\right)^{T} & \mathbf{k}_{v v}^{\mathrm{P}} & \mathbf{k}_{v w_{b}}^{\mathrm{P}} & \mathbf{k}_{v w_{s}}^{\mathrm{P}} & \mathbf{k}_{v w_{z}}^{\mathrm{P}} \\
\left(\mathbf{k}_{u w_{b}}^{\mathrm{P}}\right)^{T} & \left(\mathbf{k}_{v w_{b}}^{\mathrm{P}}\right)^{T} & \mathbf{k}_{w_{b} w_{b}}^{\mathrm{P}} & \mathbf{k}_{w_{b} w_{s}}^{\mathrm{P}} & \mathbf{k}_{w_{b} w_{z}}^{\mathrm{P}} \\
\left(\mathbf{k}_{u w_{s}}^{\mathrm{P}}\right)^{T} & \left(\mathbf{k}_{v w_{s}}^{\mathrm{P}}\right)^{T} & \left(\mathbf{k}_{w_{b} w_{s}}^{\mathrm{P}}\right)^{T} & \mathbf{k}_{w_{s} w_{s}}^{\mathrm{P}} & \mathbf{k}_{w_{s} w_{z}}^{\mathrm{P}} \\
\left(\mathbf{k}_{u w_{z}}^{\mathrm{P}}\right)^{T} & \left(\mathbf{k}_{v w_{z}}^{\mathrm{P}}\right)^{T} & \left(\mathbf{k}_{w_{b} w_{z}}^{\mathrm{P}}\right)^{T} & \left(\mathbf{k}_{w_{s} w_{z}}^{\mathrm{P}}\right)^{T} & \mathbf{k}_{w_{z} w_{z}}^{\mathrm{P}}
\end{array}\right],
$$

where the sub-matrices have the following forms

$$
\underset{4 \times 4}{\mathbf{k}_{u u}^{\mathrm{P}}}=\int_{-1}^{1} \int_{-1}^{1}\left(\mathbf{N}_{, x}^{T} \mathrm{~A}_{11} \mathbf{N}_{, x}+\mathbf{N}_{, y}^{T} \mathrm{C}_{11} \mathbf{N}_{, y}\right)|\mathbf{J}| \mathrm{d} \xi \mathrm{d} \eta,
$$




$$
\begin{aligned}
& \mathbf{k}_{4 \times 4}^{\mathrm{P}}=\int_{-1}^{1} \int_{-1}^{1}\left(\mathbf{N}_{, y}^{T} \mathrm{~A}_{11} \mathbf{N}_{, y}+\mathbf{N}_{, x}^{T} \mathrm{C}_{11} \mathbf{N}_{, x}\right)|\mathbf{J}| \mathrm{d} \xi \mathrm{d} \eta \\
& \mathbf{k}_{16 \times 16}^{\mathrm{P}} \mathrm{w}_{b} w_{b}=\int_{-1}^{1} \int_{-1}^{1}\left[\left(\mathbf{H}_{, x x}^{T} \mathrm{~A}_{22} \mathbf{H}_{, x x}+\mathbf{H}_{, y y}^{T} \mathrm{~A}_{22} \mathbf{H}_{, y y}++2 \mathbf{H}_{, x x}^{T} \mathrm{~B}_{22} \mathbf{H}_{, y y}+4 \mathbf{H}_{, x y}^{T} \mathbf{C}_{22} \mathbf{H}_{, x y}\right)\right]|\mathbf{J}| \mathrm{d} \xi \mathrm{d} \eta, \\
& \underset{\substack{\mathbf{k}_{s} w_{s} \\
16 \times 16}}{\mathrm{P}}=\frac{1}{9 h^{4}} \int_{-1}^{1} \int_{-1}^{1}\left[16\left(\mathbf{H}_{, x x}^{T} \mathrm{~A}_{66} \mathbf{H}_{, x x}+\mathbf{H}_{, y y}^{T} \mathrm{~A}_{66} \mathbf{H}_{j, y y}\right)+32 \mathbf{H}_{, x x}^{T} \mathrm{~B}_{66} \mathbf{H}_{, y y}+64 \mathbf{H}_{, x y}^{T} \mathrm{C}_{66} \mathbf{H}_{, x y}\right. \\
& \left.+144 \mathrm{C}_{44}\left(\mathbf{H}_{, x}^{T} \mathbf{H}_{, x}+\mathbf{H}_{, y}^{T} \mathbf{H}_{, y}\right)+\left(9 h^{4} \mathrm{C}_{11}-72 h^{2} \mathrm{C}_{22}\right)\left(\mathbf{H}_{, x}^{T} \mathbf{H}_{, x}+\mathbf{H}_{, y}^{T} \mathbf{H}_{, y}\right)\right]|\mathbf{J}| \mathrm{d} \xi \mathrm{d} \eta, \\
& \mathbf{k}_{\substack{w_{z} w_{z} \\
4 \times 4}}^{\mathrm{P}}=\frac{1}{h^{4}} \int_{-1}^{1} \int_{-1}^{1}\left[\left(16 \mathrm{C}_{44}-8 h^{2} \mathrm{C}_{22}+h^{4} \mathrm{C}_{11}\right)\left(\mathbf{N}_{, x}^{T} A_{11} \mathbf{N}_{, x}+\mathbf{N}_{, y}^{T} A_{11} \mathbf{N}_{, y}\right)+64 \mathbf{N}^{T} \mathrm{~A}_{22} \mathbf{N}\right]|\mathbf{J}| \mathrm{d} \xi \mathrm{d} \eta, \\
& \mathbf{k}_{4 \times 4}^{\mathrm{P}}=\int_{-1}^{1} \int_{-1}^{1}\left(\mathbf{N}_{, x}^{T} \mathrm{~B}_{11} \mathbf{N}_{, y}+\mathbf{N}_{, y}^{T} \mathrm{C}_{11} \mathbf{N}_{, y}\right)|\mathbf{J}| \mathrm{d} \xi \mathrm{d} \eta \\
& \underset{4 \times 16}{\mathbf{k}_{u w_{b}}^{\mathrm{P}}}=-\int_{-1}^{1} \int_{-1}^{1}\left(\mathbf{N}_{, x}^{T} \mathrm{~A}_{12} \mathbf{H}_{, x x}+\mathbf{N}_{, x}^{T} \mathrm{~B}_{12} \mathbf{H}_{, y y}+2 \mathbf{N}_{, y}^{T} \mathrm{C}_{12} \mathbf{H}_{, x y}\right)|\mathbf{J}| \mathrm{d} \xi \mathrm{d} \eta, \\
& \underset{4 \times 16}{\mathbf{k}_{u w_{s}}^{\mathrm{P}}}=-\frac{4}{3 h^{2}} \int_{-1}^{1} \int_{-1}^{1}\left(\mathbf{N}_{, x}^{T} \mathrm{~A}_{23} \mathbf{H}_{, x x}+\mathbf{N}_{, x}^{T} \mathrm{~B}_{23} \mathbf{H}_{, y y}+2 \mathbf{N}_{, y}^{T} \mathrm{C}_{23} \mathbf{H}_{, x y}\right)|\mathbf{J}| \mathrm{d} \xi \mathrm{d} \eta, \\
& \mathbf{k}_{4 \times 4}^{\mathrm{P}}=-\frac{8}{h^{2}} \int_{-1}^{1} \int_{-1}^{1}\left(\mathbf{N}^{T} \mathrm{~B}_{12} \mathbf{N}_{, x}\right)|\mathbf{J}| \mathrm{d} \xi \mathrm{d} \eta, \\
& \mathbf{k}_{v \times 16}^{\mathrm{P}}=-\int_{-1}^{1} \int_{-1}^{1}\left(\mathbf{N}_{, y}^{T} \mathrm{~A}_{12} \mathbf{H}_{, y y}+\mathbf{N}_{, y}^{T} \mathrm{~B}_{12} \mathbf{H}_{, x x}+2 \mathbf{N}_{, x}^{T} \mathrm{C}_{12} \mathbf{H}_{, x y}\right)|\mathbf{J}| \mathrm{d} \xi \mathrm{d} \eta, \\
& \mathbf{k}_{v \times 16}^{\mathrm{P}}=-\frac{4}{3 h^{2}} \int_{-1}^{1} \int_{-1}^{1}\left(\mathbf{N}_{, y}^{T} \mathrm{~A}_{23} \mathbf{H}_{, y y}+\mathbf{N}_{, y}^{T} \mathrm{~B}_{23} \mathbf{H}_{, x x}+2 \mathbf{N}_{, x}^{T} \mathrm{C}_{23} \mathbf{H}_{, x y}\right)|\mathbf{J}| \mathrm{d} \xi \mathrm{d} \eta, \\
& \mathbf{k}_{v \times 4}^{\mathrm{P}}=-\frac{8}{h^{2}} \int_{-1}^{1} \int_{-1}^{1}\left(\mathbf{N}^{T} \mathrm{~B}_{12} \mathbf{N}_{, y}\right)|\mathbf{J}| \mathrm{d} \xi \mathrm{d} \eta, \\
& \underset{16 \times 16}{\mathbf{k}_{w_{b} w_{s}}^{\mathrm{P}}}=\frac{4}{3 h^{2}} \int_{-1}^{1} \int_{-1}^{1}\left(\mathbf{H}_{, x x}^{T} \mathrm{~A}_{44} \mathbf{H}_{, x x}+\mathbf{H}_{, y y}^{T} \mathrm{~A}_{44} \mathbf{H}_{, y y}+2 \mathbf{H}_{, x x}^{T} \mathrm{~B}_{44} \mathbf{H}_{, y y}+4 \mathbf{H}_{, x y}^{T} \mathrm{C}_{44} \mathbf{H}_{, y y}\right)|\mathbf{J}| \mathrm{d} \xi \mathrm{d} \eta, \\
& \underset{\substack{w_{b} w_{z} \\
16 \times 4}}{\mathrm{P}}=\frac{8}{h^{2}} \int_{-1}^{1} \int_{-1}^{1}\left(\mathbf{H}_{, x x}^{T} \mathrm{~B}_{22} \mathbf{N}+\mathbf{H}_{, y y}^{T} \mathrm{~B}_{22} \mathbf{N}\right)|\mathbf{J}| \mathrm{d} \xi \mathrm{d} \eta, \\
& \underset{16 \times 4}{\mathbf{k}_{w_{b} w_{z}}^{\mathrm{P}}}=\frac{1}{3 h^{4}} \int_{-1}^{1} \int_{-1}^{1}\left[32\left(\mathbf{H}_{, x x}^{T} B_{44} \mathbf{N}+\mathbf{H}_{, y y}^{T} B_{44} \mathbf{N}\right)-24 h^{2}\left(\mathbf{H}_{, x}^{T} \mathrm{C}_{22} \mathbf{N}_{, x}+\mathbf{H}_{, y}^{T} \mathrm{C}_{22} \mathbf{N}_{, y}\right)\right. \\
& +\int_{-1}^{1} \int_{-1}^{1} \mathbf{k}_{16 \times 4}^{\mathrm{P}} w_{b} w_{z}=\frac{1}{3 h^{4}}\left[+\left(3 h^{4} \mathrm{C}_{11}+48 \mathrm{~B}_{44}\right)\left(\mathbf{H}_{, x}^{T} \mathbf{N}_{, x}+\mathbf{H}_{, y}^{T} \mathbf{N}_{, y}\right)\right]|\mathbf{J}| \mathrm{d} \xi \mathrm{d} \eta,
\end{aligned}
$$


with $|\mathbf{J}|=\operatorname{det}(\mathbf{J})$.

The element stiffness matrix stemming from the foundation deformation is of the form

$$
\mathbf{k}^{\mathrm{F}}=\left[\begin{array}{ccccc}
\mathbf{0} & \mathbf{0} & \mathbf{0} & \mathbf{0} & \mathbf{0} \\
\mathbf{0} & \mathbf{0} & \mathbf{0} & \mathbf{0} & \mathbf{0} \\
\mathbf{0} & \mathbf{0} & \mathbf{k}_{w_{b} w_{b}}^{\mathrm{F}} & \mathbf{k}_{w_{b} w_{s}}^{\mathrm{F}} & \mathbf{k}_{w_{b} w_{z}}^{\mathrm{F}} \\
\mathbf{0} & \mathbf{0} & \left(\mathbf{k}_{w_{b} w_{s}}^{\mathrm{F}}\right)^{T} & \mathbf{k}_{w_{s}}^{\mathrm{F}} w_{s} & \mathbf{k}_{w_{s} w_{z}}^{\mathrm{F}} \\
\mathbf{0} & \mathbf{0} & \left(\mathbf{k}_{w_{b} w_{z}}^{\mathrm{F}}\right)^{T} & \left(\mathbf{k}_{w_{s} w_{z}}^{\mathrm{F}}\right)^{T} & \mathbf{k}_{w_{z} w_{z}}^{\mathrm{F}}
\end{array}\right],
$$

where

$$
\begin{aligned}
& \underset{\substack{\mathbf{k}_{b} w_{b} \\
16 \times 16}}{\mathrm{~F}}=\underset{\substack{\mathbf{k}_{w_{s}} w_{s} \\
16 \times 16}}{\mathrm{~F}}=\underset{\substack{\mathbf{k}_{w_{b}} w_{s} \\
w_{16 \times 16}}}{\mathrm{~F}}=\int_{-1}^{1} \int_{-1}^{1}\left(\mathbf{H}^{T} k_{0} \mathbf{H}+\mathbf{H}_{, x}^{T} k_{1} \mathbf{H}_{, x}+\mathbf{H}_{, y}^{T} k_{1} \mathbf{H}_{, y}\right)|\mathbf{J}| \mathrm{d} \xi \mathrm{d} \eta, \\
& \mathbf{k}_{\substack{w_{z} w_{z} \\
\mathrm{~F}}}^{\mathrm{F}}=\int_{-1}^{1} \int_{-1}^{1}\left(\mathbf{N}^{T} k_{0} \mathbf{N}+\mathbf{N}_{, x}^{T} k_{1} \mathbf{N}_{, x}+\mathbf{N}_{, y}^{T} k_{1} \mathbf{N}_{, y}\right)|\mathbf{J}| \mathrm{d} \xi \mathrm{d} \eta, \\
& \underset{16 \times 4}{\mathbf{k}_{w_{b} w_{z}}^{\mathrm{F}}}=\underset{16 \times 4}{\mathbf{k}_{w_{s} w_{z}}^{\mathrm{F}}}=\int_{-1}^{1} \int_{-1}^{1}\left(\mathbf{H}^{T} k_{0} \mathbf{N}+\mathbf{H}_{, x}^{T} k_{1} \mathbf{N}_{, x}+\mathbf{H}_{, y}^{T} k_{1} \mathbf{N}_{, y}\right)|\mathbf{J}| \mathrm{d} \xi \mathrm{d} \eta .
\end{aligned}
$$

Similarly, the kinetic energy can be written in the following form

$$
T=\frac{1}{2} \sum_{i}^{N E_{p}} \dot{\mathbf{d}}_{i}^{T} \mathbf{m}_{i} \dot{\mathbf{d}}_{i},
$$

where $\dot{\mathbf{d}}=\mathbf{d}, t$, and the element mass matrix $\mathbf{m}$ is defined as

$$
\mathbf{m}=\left[\begin{array}{ccccc}
\mathbf{m}_{u u} & \mathbf{0} & \mathbf{m}_{u w_{b}} & \mathbf{m}_{u w_{s}} & \mathbf{0} \\
\mathbf{0} & \mathbf{m}_{v v} & \mathbf{m}_{v w_{b}} & \mathbf{m}_{v w_{s}} & \mathbf{0} \\
\mathbf{m}_{u w_{b}}^{T} & \mathbf{m}_{v w_{b}}^{T} & \mathbf{m}_{w_{b} w_{b}} & \mathbf{m}_{w_{b} w_{s}} & \mathbf{m}_{w_{b} w_{z}} \\
\mathbf{m}_{u w_{s}}^{T} & \mathbf{m}_{v w_{s}}^{T} & \mathbf{m}_{w_{b} w_{s}}^{T} & \mathbf{m}_{w_{s} w_{s}} & \mathbf{m}_{w_{s} w_{z}} \\
\mathbf{0} & \mathbf{0} & \mathbf{m}_{w_{b} w_{z}}^{T} & \mathbf{m}_{w_{s} w_{z}}^{T} & \mathbf{m}_{w_{z} w_{z}}
\end{array}\right]
$$

where the sub-matrices have the following forms

$$
\begin{aligned}
& \underset{\substack{u \times 4 \\
\mathbf{m}_{u u}}}{ }=\mathbf{m}_{4 \times 4}=\int_{-1}^{1} \int_{-1}^{1} \mathbf{N}^{T} \mathrm{I}_{11} \mathbf{N}|\mathbf{J}| \mathrm{d} \xi \mathrm{d} \eta, \\
& \underset{\substack{\mathbf{m}_{b} w_{b} \\
16 \times 16}}{\mathbf{m}_{-1}}=\int_{-1}^{1} \int_{-1}^{1}\left(\left(\mathbf{H}^{T} \mathrm{I}_{11} \mathbf{H}+\mathbf{H}_{, x}^{T} \mathrm{I}_{22} \mathbf{H}_{, x}+\mathbf{H}_{, y}^{T} \mathrm{I}_{22} \mathbf{H}_{, y}\right)\right)|\mathbf{J}| \mathrm{d} \xi \mathrm{d} \eta,
\end{aligned}
$$




$$
\begin{aligned}
& \mathbf{m}_{w_{s} w_{s}}=\int_{-1}^{1} \int_{-1}^{1}\left(\mathbf{H}^{T} \mathrm{I}_{11} \mathbf{H}+\frac{16}{9 h^{4}}\left(\mathbf{H}_{, x}^{T} \mathrm{I}_{66} \mathbf{H}_{, x}+\mathbf{H}_{, y}^{T} \mathrm{I}_{66} \mathbf{H}_{, y}\right)\right)|\mathbf{J}| \mathrm{d} \xi \mathrm{d} \eta, \\
& \mathbf{m}_{w_{z} w_{z}}=\int_{-1}^{1} \int_{-1}^{1}\left(\mathbf{N}^{T} \mathrm{I}_{11} \mathbf{N}-\frac{8}{h^{2}} \mathbf{N}^{T} \mathrm{I}_{22} \mathbf{N}+\frac{16}{h^{4}} \mathbf{N}^{T} \mathrm{I}_{44} \mathbf{N}\right)|\mathbf{J}| \mathrm{d} \xi \mathrm{d} \eta, \\
& \mathbf{m}_{u w_{b}}=-\int_{-1}^{1} \int_{-1}^{1}\left(\mathbf{N}^{T} \mathrm{I}_{12} \mathbf{H}_{, x}\right)|\mathbf{J}| \mathrm{d} \xi \mathrm{d} \eta, \\
& \mathbf{m}_{u w_{s}}=-\frac{4}{3 h^{2}} \int_{-1}^{1} \int_{-1}^{1}\left(\mathbf{N}^{T} \mathrm{I}_{23} \mathbf{H}_{, x}\right)|\mathbf{J}| \mathrm{d} \xi \mathrm{d} \eta, \\
& \mathbf{m}_{v w_{b}}=-\int_{-1}^{1} \int_{-1}^{1}\left(\mathbf{N}^{T} \mathrm{I}_{12} \mathbf{H}_{, y}\right)|\mathbf{J}| \mathrm{d} \xi \mathrm{d} \eta, \\
& 4 \times 16 \\
& \mathbf{m}_{v w_{s}}=-\frac{4}{3 h^{2}} \int_{-1}^{1} \int_{-1}^{1}\left(\mathbf{N}^{T} \mathrm{I}_{23} \mathbf{H}_{, y}\right)|\mathbf{J}| \mathrm{d} \xi \mathrm{d} \eta, \\
& \mathbf{m}_{w_{b} w_{s}}=\int_{-1}^{1} \int_{-1}^{1}\left(\mathbf{H}^{T} \mathrm{I}_{11} \mathbf{H}_{16}+\frac{4}{3 h^{2}}\left(\mathbf{H}_{, x}^{T} \mathrm{I}_{44} \mathbf{H}_{, x}+\mathbf{H}_{, y}^{T} \mathrm{I}_{44} \mathbf{H}_{, y}\right)\right)|\mathbf{J}| \mathrm{d} \xi \mathrm{d} \eta .
\end{aligned}
$$

Since the highest order of the polynomials under the integrals in Eqs. (33) and (36) is six, and thus 4-Gauss point along the $\xi$ and $\eta$ directions is enough to evaluate the integrals.

Having the derived element stiffness and mass matrices, the equation of motion for free vibration analysis of the plate can be written in the following form

$$
\mathbf{M D}+\mathbf{K D}=\mathbf{0},
$$

where $\mathbf{D}$ and $\ddot{\mathbf{D}}$ are, respectively, the structural vectors of nodal displacements and accelerations; $\mathbf{M}$ and $\mathbf{K}$ are the structural mass and stiffness matrices of the plate-elastic foundation system, obtained by assembling the above derived element mass and stiffness matrices, respectively.

For free vibration problems, Eq. (38) can be expressed as the following eigenvalue problem, which can be solved in the standard way to obtain natural frequencies and mode shapes of the plate

$$
\left([\mathbf{K}]-\omega^{2}[\mathbf{M}]\right)\{\mathbf{X}\}=\mathbf{0},
$$

where $\omega$ is the eigenfrequency, $\{\mathbf{X}\}$ is the generalized eigenvector. 


\section{NUMERICAL RESULTS AND DISCUSSION}

The vibration characteristics of the FGSW plate partially supported by the elastic foundation are reported in this section. Otherwise stated, the plate formed from Alumina $\left(\mathrm{Al}_{2} \mathrm{O}_{3}\right)$ and Aluminum ( $\left.\mathrm{Al}\right)$ with the following properties [25] is employed in the analysis

- Alumina $\mathrm{Al}_{2} \mathrm{O}_{3}$ (ceramic): $E_{c}=380 \mathrm{GPa} ; v_{c}=0.3 ; \rho_{c}=3800 \mathrm{~kg} / \mathrm{m}^{3}$.

- Aluminum (Al) (metal): $E_{m}=70 \mathrm{GPa} ; v_{m}=0.3 ; \rho_{m}=2707 \mathrm{~kg} / \mathrm{m}^{3}$.

For convenience of discussion, the following non-dimensional frequency parameter and foundation stiffness parameters are used [25]

$$
\bar{\omega}=\left(\omega a^{2} / h\right) \sqrt{\rho_{0} / E_{0}}, \quad K_{w}=k_{0} a^{4} / D_{C}, \quad K_{s}=k_{1} a^{2} / D_{C},
$$

where $\omega$ is the fundamental frequency, and $D_{C}=E_{c} h^{3} /\left[12\left(1-v^{2}\right)\right], E_{0}=1 \mathrm{GPa}, \rho_{0}=$ $1 \mathrm{~kg} / \mathrm{m}^{3}$. Three number in brackets are used herein to denote the layer thickness ratio, e.g. (1-2-1) means that the thickness ratio of the bottom layer, the core layer and the top layer is 1:2:1. Three types of boundary conditions, namely simply supported at all edges (SSSS), simply supported at two opposite edges and clamped at the others (SCSC) and clamped at all edges (CCCC), are considered herein. The constraints for these boundaries are as follows:

- For simply supported edge:

$$
\begin{aligned}
& \quad+u_{0}=w_{b}=w_{s}=w_{z}=w_{b, y}=w_{s, y}=w_{b, x y}=w_{s, x y}=0 \text { at } x=0, a . \\
& \quad+v_{0}=w_{b}=w_{s}=w_{z}=w_{b, x}=w_{s, x}=w_{b, x y}=w_{s, x y}=0 \text { at } y=0, b . \\
& \text { - Clamped egde: } u_{0}=v_{0}=w_{b}=w_{s}=w_{z}=w_{b, x}=w_{b, y}=w_{s, x}=w_{s, y}=w_{b, x y}=
\end{aligned}
$$
$w_{s, x y}=0$.

\subsection{Formulation verification}

Since the data for the FGSW plate partially supported by the elastic foundation are not available in the literature, the verification is carried out herewith by comparing the frequency parameters obtained in the present work with the published data as shown in Tab. 1 for a simply supported FGSW plate fully supported by the elastic foundation. For both the side-to-thickness ratios, Tab. 1 shows a good agreement between the result of the present work with that of Ref. [25], regardless of the material grading indexes, the foundation stiffness parameters and the layer thickness ratio. Noting that the plate used to obtain the result in Tab. 1 is formed from Aluminum and Zirconia as employed in [25]. In addition, the convergence of the present formulation in evaluating the frequencies in Tab. 1 has been achieved by using 20 elements, and this number of elements is used in all computations reported below.

\subsection{Simply supported plate}

The frequency parameters of the SSSS square FGSW plate partially resting on the elastic foundation are respectively listed in Tabs. 2, 3 and 4 for different values of the foundation stiffness parameters, the layer thickness ratio, and different foundation supporting areas, namely $\left(a_{f}, b_{f}\right)=(a / 4, b / 4),\left(a_{f}, b_{f}\right)=(a / 2, b / 2)$ and $\left(a_{f}, b_{f}\right)=(3 a / 4,3 b / 4)$. As in case of the plate without or fully foundation support, the frequency parameter in the table shows a decrease by the increase of the material grading indexes, regardless 
Table 1. Comparison of frequency parameter of simply SSSS square plate fully supported by elastic foundation

\begin{tabular}{|c|c|c|c|c|c|c|c|c|c|}
\hline$a / h$ & $N$ & $K_{w}$ & $K_{s}$ & Theory & $(2-1-2)$ & $(1-1-1)$ & $(2-2-1)$ & $(1-2-1)$ & $(1-0-1)$ \\
\hline \multirow{18}{*}{5} & \multirow{6}{*}{0} & \multirow{2}{*}{0} & \multirow{2}{*}{0} & Akavci [25] & 1.1912 & 1.1912 & 1.1912 & 1.1912 & 1.1912 \\
\hline & & & & Present work & 1.1985 & 1.1985 & 1.1985 & 1.1985 & 1.1985 \\
\hline & & \multirow{2}{*}{10} & \multirow{2}{*}{10} & Akavci [25] & 1.5135 & 1.5135 & 1.5135 & 1.5135 & 1.5135 \\
\hline & & & & Present work & 1.5400 & 1.5400 & 1.5400 & 1.5400 & 1.5400 \\
\hline & & \multirow{2}{*}{100} & \multirow{2}{*}{100} & Akavci [25] & 3.0908 & 3.0908 & 3.0908 & 3.0908 & 3.0908 \\
\hline & & & & Present work & 3.0980 & 3.0980 & 3.0980 & 3.0980 & 3.0980 \\
\hline & \multirow{6}{*}{2} & \multirow{2}{*}{0} & \multirow{2}{*}{0} & Akavci [25] & 0.9318 & 0.9541 & 0.9755 & 0.9927 & 0.9088 \\
\hline & & & & Present work & 0.9211 & 0.9433 & 0.9682 & 0.9832 & 0.8993 \\
\hline & & \multirow{2}{*}{10} & \multirow{2}{*}{10} & Akavci [25] & 1.3341 & 1.3469 & 1.3611 & 1.3713 & 1.3231 \\
\hline & & & & Present work & 1.3531 & 1.3650 & 1.3809 & 1.3892 & 1.3437 \\
\hline & & \multirow{2}{*}{100} & \multirow{2}{*}{100} & Akavci [25] & 2.6823 & 2.7579 & 2.7937 & 2.8476 & 2.5621 \\
\hline & & & & Present work & 2.6425 & 2.7274 & 2.7667 & 2.8278 & 2.5068 \\
\hline & \multirow{6}{*}{10} & \multirow{2}{*}{0} & \multirow{2}{*}{0} & Akavci [25] & 0.8791 & 0.8969 & 0.9215 & 0.9356 & 0.8633 \\
\hline & & & & Present work & 0.8818 & 0.8992 & 0.9270 & 0.9379 & 0.8659 \\
\hline & & \multirow{2}{*}{10} & \multirow{2}{*}{10} & Akavci [25] & 1.3045 & 1.3119 & 1.3274 & 1.3339 & 1.3022 \\
\hline & & & & Present work & 1.3343 & 1.3410 & 1.3576 & 1.3617 & 1.3320 \\
\hline & & \multirow{2}{*}{100} & \multirow{2}{*}{100} & Akavci [25] & 2.5044 & 2.6178 & 2.6707 & 2.7495 & 2.3176 \\
\hline & & & & Present work & 2.4944 & 2.6115 & 2.6647 & 2.7471 & 2.3013 \\
\hline \multirow{18}{*}{100} & \multirow{6}{*}{0} & \multirow{2}{*}{0} & 0 & Akavci [25] & 1.3404 & 1.3404 & 1.3404 & 1.3404 & 1.3404 \\
\hline & & & 0 & Present work & 1.3512 & 1.3512 & 1.3512 & 1.3512 & 1.3512 \\
\hline & & 10 & & Akavci [25] & 1.6590 & 1.6590 & 1.6590 & 1.6590 & 1.6590 \\
\hline & & 10 & 10 & Present work & 1.6678 & 1.6678 & 1.6678 & 1.6678 & 1.6678 \\
\hline & & 100 & & Akavci [25] & 3.3694 & 3.3694 & 3.3694 & 3.3694 & 3.3694 \\
\hline & & 100 & 100 & Present work & 3.3740 & 3.3740 & 3.3740 & 3.3740 & 3.3740 \\
\hline & & 0 & 0 & Akavci [25] & 1.0182 & 1.0428 & 1.0694 & 1.0885 & 0.9971 \\
\hline & & 0 & 0 & Present work & 1.0076 & 1.0311 & 1.0620 & 1.0778 & 0.9898 \\
\hline & & 10 & 10 & Akavci [25] & 1.43 & 1.4444 & 1.4623 & 1.4740 & 1.4200 \\
\hline & 2 & 10 & 10 & Present work & 1.4225 & 1.4361 & 1.4569 & 1.4662 & 1.4149 \\
\hline & & 100 & 100 & Akavci [25] & 3.3344 & 3.3283 & 3.3300 & 3.3261 & 3.3491 \\
\hline & & 100 & 100 & Present work & 3.3315 & 3.3250 & 3.3279 & 3.3229 & 3.3472 \\
\hline & & 0 & 0 & Akavci [25] & 0.9602 & 0.9758 & 1.0062 & 1.0191 & 0.9580 \\
\hline & & 0 & 0 & Present work & 0.9657 & 0.9802 & 1.0143 & 1.0228 & 0.9651 \\
\hline & 10 & 10 & & Akavci [25] & 1.3967 & 1.4029 & 1.4219 & 1.4278 & 1.4023 \\
\hline & 10 & 10 & 10 & Present work & 1.4005 & 1.4060 & 1.4277 & 1.4305 & 1.4073 \\
\hline & & 100 & 100 & $\mathrm{Ak}_{\tilde{z}}$ & 3.3480 & 3.3332 & 3.3327 & 3.3225 & 3.3772 \\
\hline & & 100 & 100 & Present work & 3.3499 & 3.3348 & 3.3354 & 3.3240 & 3.3795 \\
\hline
\end{tabular}


Free vibration of FG sandwich plates partially supported by elastic foundation using a quasi-3D finite element formulation 77

Table 2. Frequency parameter of SSSS square plates partially supported by elastic foundation with $\left(a_{f}, b_{f}\right)=(a / 4, b / 4)$

\begin{tabular}{|c|c|c|c|c|c|c|c|c|c|}
\hline$a / h$ & $N$ & $K_{w}$ & $K_{s}$ & $(2-1-2)$ & $(1-1-1)$ & $(2-2-1)$ & $(1-2-1)$ & $(1-0-1)$ & $(1-8-1)$ \\
\hline \multirow{9}{*}{5} & \multirow{3}{*}{0.5} & 0 & 0 & 1.1886 & 1.2389 & 1.2867 & 1.3208 & 1.1343 & 1.5215 \\
\hline & & 10 & 10 & 1.2349 & 1.2833 & 1.3294 & 1.3625 & 1.1830 & 1.5577 \\
\hline & & 100 & 100 & 1.3905 & 1.4408 & 1.4865 & 1.5211 & 1.3344 & 1.7146 \\
\hline & \multirow{3}{*}{2} & 0 & 0 & 0.9637 & 1.0241 & 1.1006 & 1.1400 & 0.9159 & 1.4431 \\
\hline & & 10 & 10 & 1.0211 & 1.0782 & 1.1510 & 1.1888 & 0.9766 & 1.4816 \\
\hline & & 100 & 100 & 1.1660 & 1.2294 & 1.3030 & 1.3461 & 1.1095 & 1.6404 \\
\hline & \multirow{3}{*}{10} & 0 & 0 & 0.8934 & 0.9396 & 1.0267 & 1.0562 & 0.8678 & 1.4033 \\
\hline & & 10 & 10 & 0.9565 & 0.9993 & 1.0814 & 1.1094 & 0.9330 & 1.4432 \\
\hline & & 100 & 100 & 1.0930 & 1.1444 & 1.2287 & 1.2643 & 1.0481 & 1.6031 \\
\hline \multirow{9}{*}{10} & \multirow{3}{*}{0.5} & 0 & 0 & 1.2590 & 1.3139 & 1.3688 & 1.4061 & 1.2033 & 1.6405 \\
\hline & & 10 & 10 & 1.3054 & 1.3581 & 1.4112 & 1.4473 & 1.2521 & 1.6757 \\
\hline & & 100 & 100 & 1.4936 & 1.5470 & 1.5992 & 1.6361 & 1.4388 & 1.8601 \\
\hline & \multirow{3}{*}{2} & 0 & 0 & 1.0096 & 1.0731 & 1.1586 & 1.1999 & 0.9666 & 1.5471 \\
\hline & & 10 & 10 & 1.0682 & 1.1280 & 1.2094 & 1.2489 & 1.0290 & 1.5849 \\
\hline & & 100 & 100 & 1.2472 & 1.3115 & 1.3936 & 1.4375 & 1.2021 & 1.7724 \\
\hline & \multirow{3}{*}{10} & 0 & 0 & 0.9351 & 0.9808 & 1.0773 & 1.1061 & 0.9295 & 1.4998 \\
\hline & & 10 & 10 & 1.0000 & 1.0418 & 1.1328 & 1.1600 & 0.9976 & 1.5391 \\
\hline & & 100 & 100 & 1.1727 & 1.2198 & 1.3128 & 1.3464 & 1.1625 & 1.7284 \\
\hline \multirow{9}{*}{100} & \multirow{3}{*}{0.5} & 0 & 0 & 1.2861 & 1.3428 & 1.4007 & 1.4393 & 1.2298 & 1.6882 \\
\hline & & 10 & 10 & 1.3325 & 1.3870 & 1.4430 & 1.4804 & 1.2787 & 1.7230 \\
\hline & & 100 & 100 & 1.5345 & 1.5890 & 1.6439 & 1.6816 & 1.4808 & 1.9186 \\
\hline & \multirow{3}{*}{2} & 0 & 0 & 1.0268 & 1.0915 & 1.1806 & 1.2225 & 0.9860 & 1.5882 \\
\hline & & 10 & 10 & 1.0858 & 1.1466 & 1.2315 & 1.2716 & 1.0489 & 1.6257 \\
\hline & & 100 & 100 & 1.2797 & 1.3439 & 1.4294 & 1.4734 & 1.2410 & 1.8251 \\
\hline & \multirow{3}{*}{10} & 0 & 0 & 0.9507 & 0.9961 & 1.0963 & 1.1248 & 0.9538 & 1.5377 \\
\hline & & 10 & 10 & 1.0163 & 1.0576 & 1.1521 & 1.1789 & 1.0230 & 1.5767 \\
\hline & & 100 & 100 & 1.2053 & 1.2498 & 1.3463 & 1.3786 & 1.2147 & 1.7781 \\
\hline
\end{tabular}

of the layer thickness ratio and the foundation stiffness. The decrease of the frequency parameter can be explained by the lower content of ceramic for the plate associated with a higher index $n$, as can be seen from Eq. (1). The tables also show an important role of the layer thickness ratio and the area of the foundation support on the frequency parameter. A larger core thickness the plate has a higher frequency is, irrespective of the foundation supporting area and the foundation stiffness parameters. The effect of the layer thickness ratio, however influenced by the foundation support also. For example, with $a / h=5, n=2,\left(K_{w}, K_{s}\right)=(10,10)$, the frequency parameter increases $31.08 \%$ when 
Table 3. Frequency parameter of SSSS square plates partially supported by elastic foundation with $\left(a_{f}, b_{f}\right)=(a / 2, b / 2)$

\begin{tabular}{|c|c|c|c|c|c|c|c|c|c|}
\hline$a / h$ & $N$ & $K_{w}$ & $K_{s}$ & $(2-1-2)$ & $(1-1-1)$ & $(2-2-1)$ & $(1-2-1)$ & $(1-0-1)$ & $(1-8-1)$ \\
\hline \multirow{9}{*}{5} & \multirow{3}{*}{0.5} & 0 & 0 & 1.1886 & 1.2389 & 1.2867 & 1.3208 & 1.1343 & 1.5215 \\
\hline & & 10 & 10 & 1.3976 & 1.4397 & 1.4810 & 1.5099 & 1.3536 & 1.6870 \\
\hline & & 100 & 100 & 2.1598 & 2.2051 & 2.2451 & 2.2754 & 2.1064 & 2.4391 \\
\hline & \multirow{3}{*}{2} & 0 & 0 & 0.9637 & 1.0241 & 1.1006 & 1.1400 & 0.9159 & 1.4431 \\
\hline & & 10 & 10 & 1.2200 & 1.2659 & 1.3286 & 1.3593 & 1.1878 & 1.6186 \\
\hline & & 100 & 100 & 1.9519 & 2.0172 & 2.0863 & 2.1278 & 1.8823 & 2.3813 \\
\hline & \multirow{3}{*}{10} & 0 & 0 & 0.8934 & 0.9396 & 1.0267 & 1.0562 & 0.8678 & 1.4033 \\
\hline & & 10 & 10 & 1.1745 & 1.2050 & 1.2741 & 1.2943 & 1.1638 & 1.5850 \\
\hline & & 100 & 100 & 1.8799 & 1.9384 & 2.0210 & 2.0585 & 1.7962 & 2.3541 \\
\hline \multirow{9}{*}{10} & \multirow{3}{*}{0.5} & 0 & 0 & 1.2590 & 1.3139 & 1.3688 & 1.4061 & 1.2033 & 1.6405 \\
\hline & & 10 & 10 & 1.4659 & 1.5121 & 1.5599 & 1.5917 & 1.4206 & 1.8004 \\
\hline & & 100 & 100 & 2.3095 & 2.3529 & 2.3967 & 2.4257 & 2.2658 & 2.6079 \\
\hline & \multirow{3}{*}{2} & 0 & 0 & 1.0096 & 1.0731 & 1.1586 & 1.1999 & 0.9666 & 1.5471 \\
\hline & & 10 & 10 & 1.2669 & 1.3151 & 1.3857 & 1.4179 & 1.2399 & 1.7179 \\
\hline & & 100 & 100 & 2.1021 & 2.1585 & 2.2322 & 2.2674 & 2.0648 & 2.5415 \\
\hline & \multirow{3}{*}{10} & 0 & 0 & 0.9351 & 0.9808 & 1.0773 & 1.1061 & 0.9295 & 1.4998 \\
\hline & & 10 & 10 & 1.2185 & 1.2478 & 1.3249 & 1.3442 & 1.2277 & 1.6772 \\
\hline & & 100 & 100 & 2.0432 & 2.0832 & 2.1704 & 2.1959 & 2.0384 & 2.5101 \\
\hline \multirow{9}{*}{100} & \multirow{3}{*}{0.5} & 0 & 0 & 1.2861 & 1.3428 & 1.4007 & 1.4393 & 1.2298 & 1.6882 \\
\hline & & 10 & 10 & 1.4924 & 1.5402 & 1.5908 & 1.6238 & 1.4466 & 1.8462 \\
\hline & & 100 & 100 & 2.3656 & 2.4083 & 2.4539 & 2.4824 & 2.3261 & 2.6736 \\
\hline & \multirow{3}{*}{2} & 0 & 0 & 1.0268 & 1.0915 & 1.1806 & 1.2225 & 0.9860 & 1.5882 \\
\hline & & 10 & 10 & 1.2845 & 1.3336 & 1.4074 & 1.4402 & 1.2598 & 1.7574 \\
\hline & & 100 & 100 & 2.1593 & 2.2114 & 2.2868 & 2.3192 & 2.1376 & 2.6028 \\
\hline & \multirow{3}{*}{10} & 0 & 0 & 0.9507 & 0.9961 & 1.0963 & 1.1248 & 0.9538 & 1.5377 \\
\hline & & 10 & 10 & 1.2350 & 1.2638 & 1.3441 & 1.3629 & 1.2531 & 1.7136 \\
\hline & & 100 & 100 & 2.1073 & 2.1383 & 2.2267 & 2.2470 & 2.1442 & 2.5694 \\
\hline
\end{tabular}

the core thickness changes from (2-1-2) to (1-8-1) for the plate supported by the foundation with $\left(a_{f}, b_{f}\right)=(a / 4, b / 4)$, while this value decreases to 24.63 and 19.06 for the plate supported by the foundation with $\left(a_{f}, b_{f}\right)=(a / 2, b / 2)$ and $\left(a_{f}, b_{f}\right)=(3 a / 4,3 b / 4)$, respectively. By comparing the frequency parameters in the three tables, one can see that the frequency parameter remarkably increases by increasing the foundation supporting area, regardless of the material grading index $n$ and the foundation stiffness parameters. The effect of the shear deformation on the frequencies of the FGSW plate partially supported by the elastic foundation can also be seen from the tables, and the frequency 
Table 4. Frequency parameter of SSSS square plates partially supported by elastic foundation with $\left(a_{f}, b_{f}\right)=(3 a / 4,3 b / 4)$

\begin{tabular}{|c|c|c|c|c|c|c|c|c|c|}
\hline$a / h$ & $N$ & $K_{w}$ & $K_{s}$ & $(2-1-2)$ & $(1-1-1)$ & $(2-2-1)$ & $(1-2-1)$ & $(1-0-1)$ & $(1-8-1)$ \\
\hline \multirow{9}{*}{5} & \multirow{3}{*}{0.5} & 0 & 0 & 1.1886 & 1.2389 & 1.2867 & 1.3208 & 1.1343 & 1.5215 \\
\hline & & 10 & 10 & 1.5946 & 1.6307 & 1.6672 & 1.6919 & 1.5578 & 1.8501 \\
\hline & & 100 & 100 & 3.0190 & 3.0446 & 3.0713 & 3.0890 & 2.9940 & 3.0190 \\
\hline & \multirow{3}{*}{2} & 0 & 0 & 0.9637 & 1.0241 & 1.1006 & 1.1400 & 0.9159 & 1.4431 \\
\hline & & 10 & 10 & 1.4497 & 1.4862 & 1.5404 & 1.5641 & 1.4269 & 1.7901 \\
\hline & & 100 & 100 & 2.9412 & 2.9604 & 2.9974 & 3.0119 & 2.8567 & 3.1692 \\
\hline & \multirow{3}{*}{10} & 0 & 0 & 0.8934 & 0.9396 & 1.0267 & 1.0562 & 0.8678 & 1.4033 \\
\hline & & 10 & 10 & 1.4204 & 1.4413 & 1.5001 & 1.5127 & 1.4176 & 1.7615 \\
\hline & & 100 & 100 & 2.9568 & 2.9544 & 2.9904 & 2.9924 & 2.4105 & 3.1564 \\
\hline \multirow{9}{*}{10} & \multirow{3}{*}{0.5} & 0 & 0 & 1.2590 & 1.3139 & 1.3688 & 1.4061 & 1.2033 & 1.2590 \\
\hline & & 10 & 10 & 1.6646 & 1.7040 & 1.7465 & 1.7736 & 1.6273 & 1.6646 \\
\hline & & 100 & 100 & 3.1780 & 3.2066 & 3.2402 & 3.2593 & 3.1541 & 3.1780 \\
\hline & \multirow{3}{*}{2} & 0 & 0 & 1.0096 & 1.0731 & 1.1586 & 1.1999 & 0.9666 & 1.5471 \\
\hline & & 10 & 10 & 1.5022 & 1.5397 & 1.6009 & 1.6253 & 1.4862 & 1.8876 \\
\hline & & 100 & 100 & 3.0758 & 3.0963 & 3.1445 & 3.1581 & 3.0847 & 3.3547 \\
\hline & \multirow{3}{*}{10} & 0 & 0 & 0.9351 & 0.9808 & 1.0773 & 1.1061 & 0.9295 & 1.4998 \\
\hline & & 10 & 10 & 1.4720 & 1.4902 & 1.5558 & 1.5664 & 1.4914 & 1.8525 \\
\hline & & 100 & 100 & 3.0900 & 3.0827 & 3.1318 & 3.1281 & 3.1446 & 3.3356 \\
\hline \multirow{9}{*}{100} & \multirow{3}{*}{0.5} & 0 & 0 & 1.2861 & 1.3428 & 1.4007 & 1.4393 & 1.2298 & 1.6882 \\
\hline & & 10 & 10 & 1.6916 & 1.7325 & 1.7775 & 1.8056 & 1.6542 & 2.0056 \\
\hline & & 100 & 100 & 3.2514 & 3.2807 & 3.3175 & 3.3368 & 3.2300 & 3.4920 \\
\hline & \multirow{3}{*}{2} & 0 & 0 & 1.0268 & 1.0915 & 1.1806 & 1.2225 & 0.9860 & 1.5882 \\
\hline & & 10 & 10 & 1.5219 & 1.5598 & 1.6238 & 1.6485 & 1.5090 & 1.9265 \\
\hline & & 100 & 100 & 3.1377 & 3.1579 & 3.2116 & 3.2242 & 3.1563 & 3.4392 \\
\hline & \multirow{3}{*}{10} & 0 & 0 & 0.9507 & 0.9961 & 1.0963 & 1.1248 & 0.9538 & 1.5377 \\
\hline & & 10 & 10 & 1.4914 & 1.5083 & 1.5768 & 1.5865 & 1.5212 & 1.8885 \\
\hline & & 100 & 100 & 3.1517 & 3.1405 & 3.1960 & 3.1892 & 3.2354 & 3.4172 \\
\hline
\end{tabular}

parameter increases by the increase of the side-to-thickness ratio. The numerical result in the tables shows the ability of the derived finite element formulation on modeling the shear deformation effect of the FGSW plate partially supported by the elastic foundation.

The effect of the foundation support on the free vibration of the SSSS plate can be also seen from Fig. 2 where the first vibration mode for the transverse displacement $w$ of the plate is shown for various values of the foundation supporting areas. The first mode shape of the plate partially supported by the foundation, as seen from the figure is asymmetrical while that of the plate fully supported by the foundation is symmetrical. 
The foundation supporting area is also plays an important role on the vibration mode of the plate, and the position at which the transverse bending displacement attains the maximum value depends on the foundation supporting area.

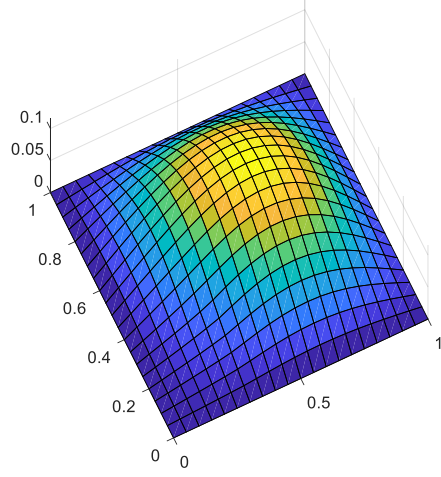

(a) $\left(a_{f}, b_{f}\right)=(a / 4, b / 4)$

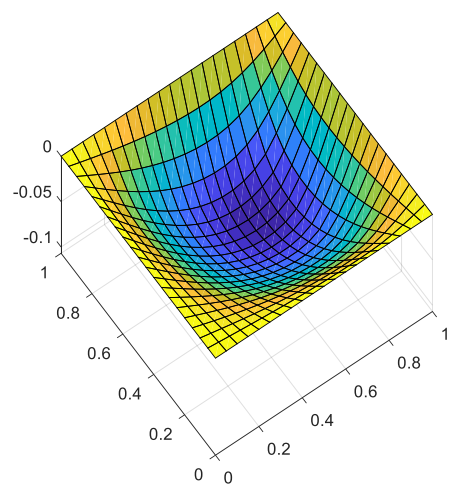

(c) $\left(a_{f}, b_{f}\right)=(3 a / 4,3 b / 4)$

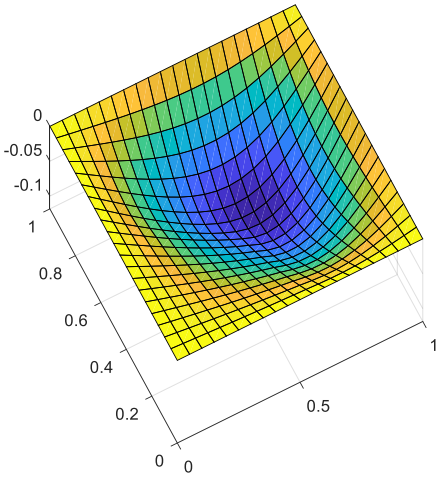

(b) $\left(a_{f}, b_{f}\right)=(a / 2, b / 2)$

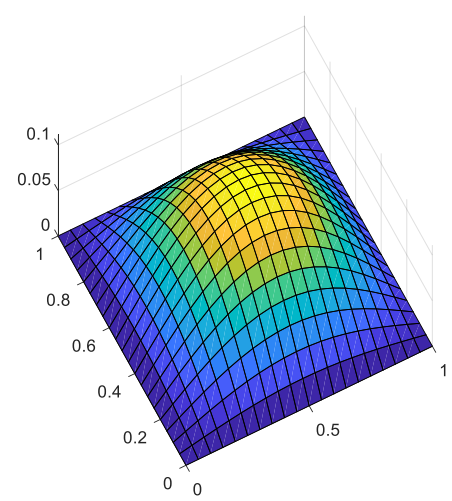

(d) $\left(a_{f}, b_{f}\right)=(a, b)$

Fig. 2. The first mode shapes for transverse displacement of SSSS (2-1-2) square plate for $a / h=10, n=2,\left(K_{w}, K_{s}\right)=(50,50)$ and different foundation supporting areas

\subsection{Plate with other boundary conditions}

The free vibration characteristics of the FGSW square plate with other boundary conditions (B.C.), namely clamped at two opposite edges and simply supported at the two others (CSCS) and clamped at all edges (CCCC), are reported in this subsection. In Tab. 5, the frequency parameters of the CSCS and CCCC plates with $a / h=10$ are listed for $\left(a_{f}, b_{f}\right)=(a / 2, b / 2)$ and various values of the foundation stiffness parameters and the layer thickness ratio. As expected, the frequency parameters of the CCCC plate are higher than the corresponding parameters of the CSCS and SSSS plates, regardless of the foundation stiffness and the layer thickness ratio. The dependence of the frequency 
parameter of the CSCS and CCCC plates upon the material grading index and the layer thickness ratio is similar to that of the SSSS plate.

Table 5. Frequency parameter of CSCS and CCCC square plates with $a / h=10$, partially supported by the foundation of $\left(a_{f}, b_{f}\right)=(a / 2, b / 2)$

\begin{tabular}{|c|c|c|c|c|c|c|c|c|c|}
\hline B.C. & $N$ & $K_{w}$ & $K_{s}$ & $(2-1-2)$ & $(1-1-1)$ & $(2-2-1)$ & $(1-2-1)$ & $(1-0-1)$ & $(1-8-1)$ \\
\hline \multirow{12}{*}{ CSCS } & \multirow{3}{*}{0.5} & 0 & 0 & 1.8227 & 1.9012 & 1.9701 & 2.0315 & 1.7409 & 2.3579 \\
\hline & & 10 & 10 & 1.9922 & 2.0630 & 2.1262 & 2.1823 & 1.9199 & 2.4868 \\
\hline & & 100 & 100 & 2.7998 & 2.8619 & 2.9177 & 2.9655 & 2.7356 & 3.2263 \\
\hline & \multirow{3}{*}{1} & 0 & 0 & 1.6163 & 1.7075 & 1.7947 & 1.8692 & 1.5332 & 2.2859 \\
\hline & & 10 & 10 & 1.8098 & 1.8896 & 1.9681 & 2.0347 & 1.7399 & 2.4196 \\
\hline & & 100 & 100 & 2.6359 & 2.7096 & 2.7818 & 2.8397 & 2.5691 & 3.1711 \\
\hline & \multirow{3}{*}{2} & 0 & 0 & 1.4693 & 1.5614 & 1.6621 & 1.7422 & 1.4025 & 2.2289 \\
\hline & & 10 & 10 & 1.6850 & 1.7627 & 1.8516 & 1.9216 & 1.6333 & 2.3669 \\
\hline & & 100 & 100 & 2.5173 & 2.5939 & 2.6799 & 2.7422 & 2.4623 & 3.1287 \\
\hline & \multirow{3}{*}{10} & 0 & 0 & 1.3622 & 1.4302 & 1.5398 & 1.6101 & 1.3405 & 2.1636 \\
\hline & & 10 & 10 & 1.6026 & 1.6550 & 1.7491 & 1.8076 & 1.5951 & 2.3072 \\
\hline & & 100 & 100 & 2.4376 & 2.4929 & 2.5903 & 2.6434 & 2.4172 & 3.0820 \\
\hline \multirow{12}{*}{ СССС } & \multirow{3}{*}{0.5} & 0 & 0 & 2.2328 & 2.3278 & 2.4078 & 2.4839 & 2.1315 & 2.8698 \\
\hline & & 10 & 10 & 2.3906 & 2.4781 & 2.5526 & 2.6234 & 2.2986 & 2.9884 \\
\hline & & 100 & 100 & 3.2398 & 3.3118 & 3.3735 & 3.4313 & 3.1639 & 3.7334 \\
\hline & \multirow{3}{*}{1} & 0 & 0 & 1.9850 & 2.0960 & 2.1970 & 2.2907 & 1.8806 & 2.7851 \\
\hline & & 10 & 10 & 2.1666 & 2.2663 & 2.3587 & 2.4445 & 2.0754 & 2.9082 \\
\hline & & 100 & 100 & 3.0572 & 3.1404 & 3.2179 & 3.2878 & 2.9796 & 3.6690 \\
\hline & \multirow{3}{*}{2} & 0 & 0 & 1.8076 & 1.9205 & 2.0365 & 2.1389 & 1.7208 & 2.7180 \\
\hline & & 10 & 10 & 2.0117 & 2.1101 & 2.2143 & 2.3065 & 1.9403 & 2.8452 \\
\hline & & 100 & 100 & 2.9295 & 3.0138 & 3.1032 & 3.1785 & 2.8649 & 3.6197 \\
\hline & \multirow{3}{*}{10} & 0 & 0 & 1.6769 & 1.7621 & 1.8873 & 1.9807 & 1.6362 & 2.6412 \\
\hline & & 10 & 10 & 1.9064 & 1.9756 & 2.0852 & 2.1663 & 1.8796 & 2.7737 \\
\hline & & 100 & 100 & 2.8478 & 2.9078 & 3.0052 & 3.0707 & 2.8090 & 3.5658 \\
\hline
\end{tabular}

Figs. 3 and 4 respectively illustrate the first four vibration modes for the transverse displacement $\mathrm{w}$ of the CSCS and CCCC plates having $a / h=10, n=2$, partially supported by the elastic foundation with $\left(a_{f}, b_{f}\right)=(a / 4, b / 4)$ and $\left(K_{w}, K_{s}\right)=(50,50)$. The influence of the foundation support on the vibration modes of the plates can be clearly seen from the figures. The symmetry of the vibration modes as seen for the plate fully supported by the foundation is destroyed by the partial foundation support. 

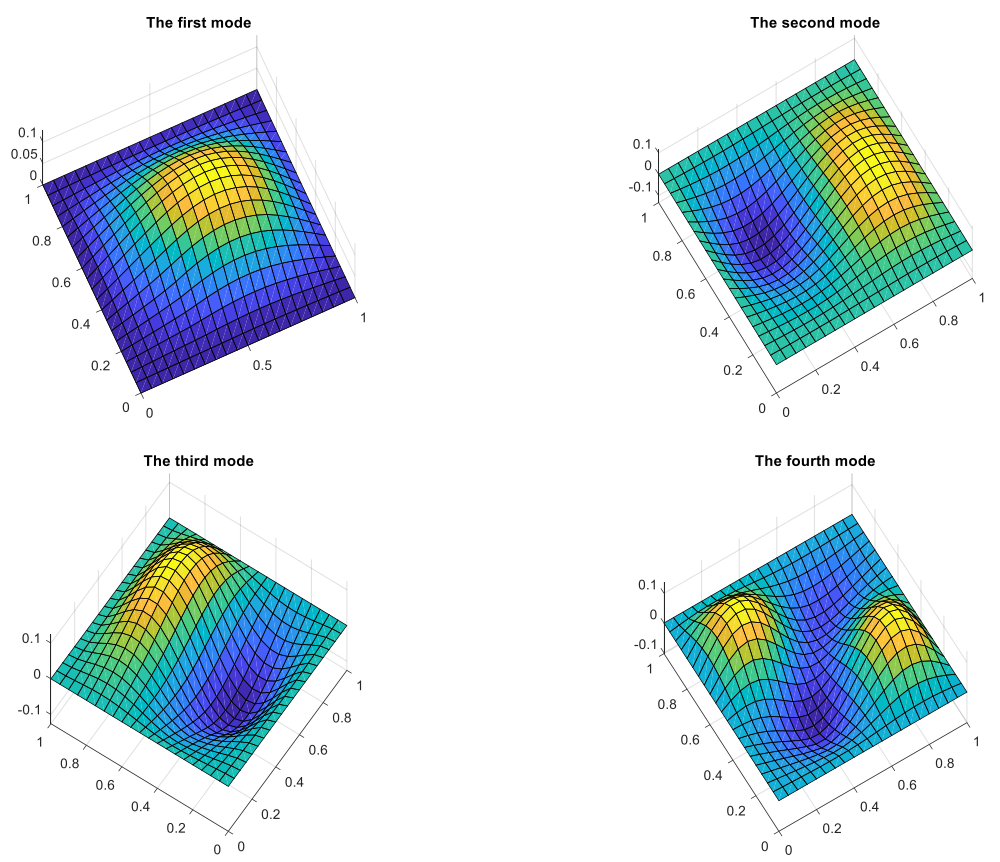

Fig. 3. The first four mode shapes for transverse displacement of CSCS (2-1-2) square plate with $a / h=10, n=2,\left(a_{f}, b_{f}\right)=(a / 4, b / 4)$ and $\left(K_{w}, K_{s}\right)=(50,50)$
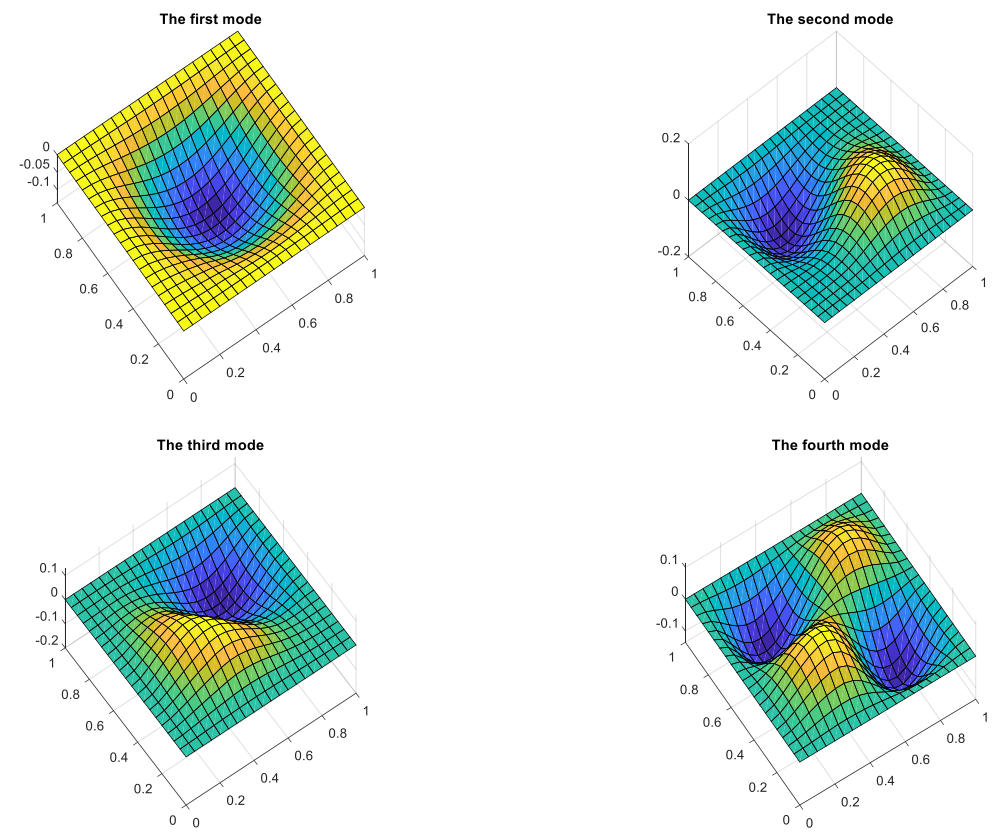

Fig. 4. The first four mode shapes for transverse displacement of CCCC (2-1-2) square plate with $a / h=10, n=2,\left(a_{f}, b_{f}\right)=(a / 4, b / 4)$ and $\left(K_{w}, K_{s}\right)=(50,50)$ 


\subsection{Plate with different side-to-thickness ratios}

The effect of the side-to-thickness ratio $a / h$ on the frequency parameter of the FGSW plate is illustrated in Fig. 5 for the (1-1-1) SSSS and CCCC square plates with $n=2$, partially supported by the elastic foundation $\left(a_{f}, b_{f}\right)=(a / 2, b / 2)$. The frequency parameter, as seen from the figure, steadily increases by increasing the aspect ratio, and the increase is the most significant for $a / h$ between 5 and 20. The foundation stiffness also plays an important role on the dependence of the frequency parameter on the aspect ratio, the increase of frequency parameter by increasing the aspect ratio is more significant when the plates are supported by the foundation with higher stiffness. The result in Fig. 5 shows again the ability of the finite element formulation derived in the present work in modeling the shear deformation effect of the FGSW plate.
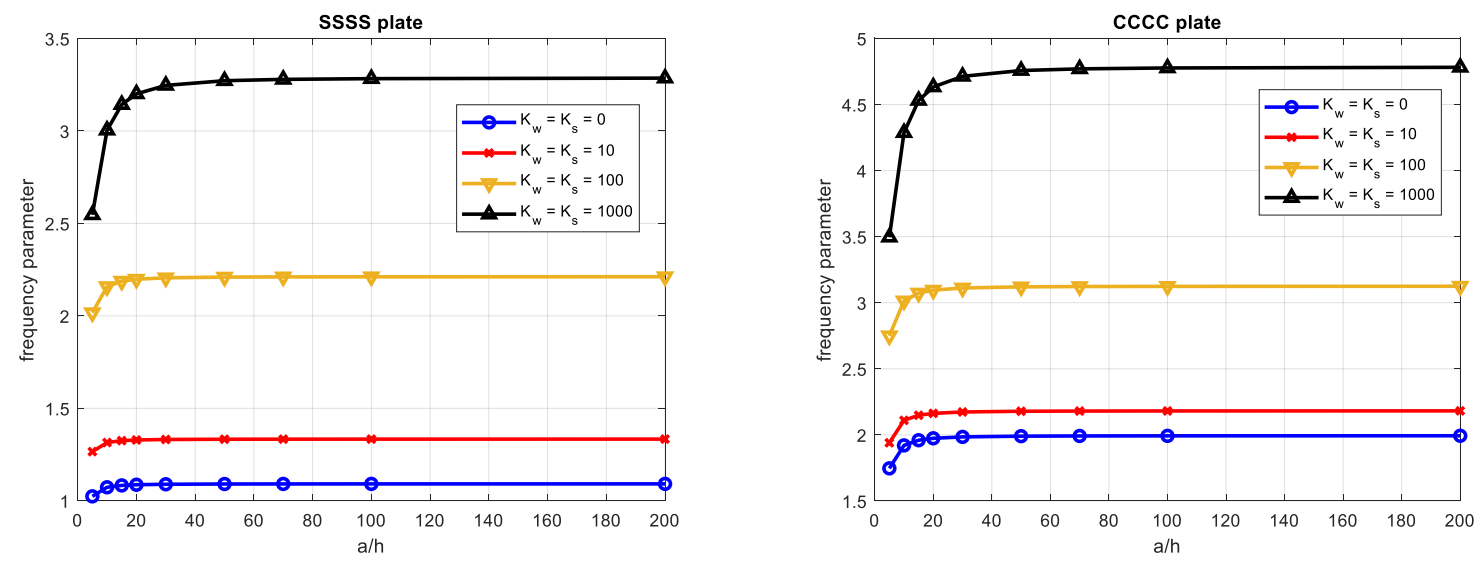

Fig. 5. Frequency parameter versus side-to-thickness ratio of (1-1-1) SSSS and CCCC square FGSW plates partially supported by elastic foundation with $\left(a_{f}, b_{f}\right)=(a / 2, b / 2),(n=2)$

\section{CONCLUSIONS}

The free vibration of FGSW plates partially supported by a Pasternak foundation has been studied using a quasi-3D finite element formulation. The plates are considered to be composed of three layers, a homogeneous ceramic core and two functionally graded skin layers. Mori-Tanaka scheme was employed to estimate the effective material properties of the plates. The frequency parameters and vibration modes have been evaluated for the FGSW plates with various boundary conditions, supported by the foundation of different areas. The numerical results obtained in the present paper reveal that the foundation supporting area plays an important role on both the frequencies and mode shapes of the plates. A parametric study has been carried out to highlight the influence of the material grading index, the layer thickness ratio and the foundation stiffness on the vibration characteristics of the plates. The effect of the side-to-thickness ratio on the frequencies of the FGSW plates has also been examined and discussed. 
Le Cong Ich, Pham Vu Nam, Nguyen Dinh Kien

\section{ACKNOWLEDGMENTS}

This work was supported by Vietnam National Foundation for Science and Technology Development (NAFOSTED) under grant number 107.02-2018.23.

\section{REFERENCES}

[1] Y. Fukui. Fundamental investigation of functionally gradient material manufacturing system using centrifugal force. Japan Society of Mechanical Engineering International Journal, Series III, 34, (1), (1991), pp. 144-148. https://doi.org/10.1299/jsmec1988.34.144.

[2] D. K. Jha, T. Kant, and R. K. Singh. A critical review of recent research on functionally graded plates. Composite Structures, 96, (2013), pp. 833-849. https://doi.org/10.1016/j.compstruct.2012.09.001.

[3] K. Swaminathan, D. T. Naveenkumar, A. M. Zenkour, and E. Carrera. Stress, vibration and buckling analyses of FGM plates-A state-of-the-art review. Composite Structures, 120, (2015), pp. 10-31. https://doi.org/10.1016/j.compstruct.2014.09.070.

[4] G. N. Praveen and J. N. Reddy. Nonlinear transient thermoelastic analysis of functionally graded ceramic-metal plates. International Journal of Solids and Structures, 35, (33), (1998), pp. 4457-4476. https://doi.org/10.1016/s0020-7683(97)00253-9.

[5] A. M. Zenkour. A comprehensive analysis of functionally graded sandwich plates: Part 1-Deflection and stresses. International journal of solids and structures, 42, (18-19), (2005), pp. 5224-5242. https://doi.org/10.1016/j.ijsolstr.2005.02.015.

[6] A. M. Zenkour. A comprehensive analysis of functionally graded sandwich plates: Part 2-Buckling and free vibration. International Journal of Solids and Structures, 42, (18-19), (2005), pp. 5243-5258. https://doi.org/10.1016/j.ijsolstr.2005.02.016.

[7] A. M. Zenkour and M. Sobhy. Thermal buckling of various types of FGM sandwich plates. Composite Structures, 93, (1), (2010), pp. 93-102. https://doi.org/10.1016/j.compstruct.2010.06.012.

[8] S. Xiang, Y.-x. Jin, Z. Bi, S. Jiang, and M. Yang. A n-order shear deformation theory for free vibration of functionally graded and composite sandwich plates. Composite Structures, 93, (11), (2011), pp. 2826-2832. https://doi.org/10.1016/j.compstruct.2011.05.022.

[9] S. Xiang, G. Kang, M. Yang, and Y. Zhao. Natural frequencies of sandwich plate with functionally graded face and homogeneous core. Composite Structures, 96, (2013), pp. 226-231. https://doi.org/10.1016/j.compstruct.2012.09.003.

[10] A. M. A. Neves, A. J. M. Ferreira, E. Carrera, M. Cinefra, C. M. C. Roque, R. M. N. Jorge, and C. M. M. Soares. Static, free vibration and buckling analysis of isotropic and sandwich functionally graded plates using a quasi-3D higher-order shear deformation theory and a meshless technique. Composites Part B: Engineering, 44, (1), (2013), pp. 657-674. https://doi.org/10.1016/j.compositesb.2012.01.089.

[11] H.-T. Thai and D.-H. Choi. Finite element formulation of various four unknown shear deformation theories for functionally graded plates. Finite Elements in Analysis and Design, 75, (2013), pp. 50-61. https://doi.org/10.1016/j.finel.2013.07.003.

[12] H.-T. Thai and S.-E. Kim. A simple higher-order shear deformation theory for bending and free vibration analysis of functionally graded plates. Composite Structures, 96, (2013), pp. 165173. https://doi.org/10.1016/j.compstruct.2012.08.025.

[13] H.-T. Thai and S.-E. Kim. A simple quasi-3D sinusoidal shear deformation theory for functionally graded plates. Composite Structures, 99, (2013), pp. 172-180. https://doi.org/10.1016/j.compstruct.2012.11.030. 
[14] H.-T. Thai, T.-K. Nguyen, T. P. Vo, and J. Lee. Analysis of functionally graded sandwich plates using a new first-order shear deformation theory. European Journal of Mechanics-A/Solids, 45, (2014), pp. 211-225. https://doi.org/10.1016/j.euromechsol.2013.12.008.

[15] L. Iurlaro, M. Gherlone, and M. Di Sciuva. Bending and free vibration analysis of functionally graded sandwich plates using the refined zigzag theory. Journal of Sandwich Structures $\mathcal{E}$ Materials, 16, (6), (2014), pp. 669-699. https://doi.org/10.1177/1099636214548618.

[16] S. Pandey and S. Pradyumna. Analysis of functionally graded sandwich plates using a higher-order layerwise theory. Composites Part B: Engineering, 153, (2018), pp. 325-336. https://doi.org/10.1016/j.compositesb.2018.08.121.

[17] Z. Belabed, A. A. Bousahla, M. S. A. Houari, A. Tounsi, and S. R. Mahmoud. A new 3-unknown hyperbolic shear deformation theory for vibration of functionally graded sandwich plate. Earthquakes and Structures, 14, (2), (2018), pp. 103-115. https://doi.org/10.12989/eas.2018.14.2.103.

[18] A. A. Daikh and A. M. Zenkour. Effect of porosity on the bending analysis of various functionally graded sandwich plates. Materials Research Express, 6, (6), (2019), p. 065703. https://doi.org/10.1088/2053-1591/ab0971.

[19] C. F. Lü, C. W. Lim, and W. Q. Chen. Exact solutions for free vibrations of functionally graded thick plates on elastic foundations. Mechanics of Advanced Materials and Structures, 16, (8), (2009), pp. 576-584. https://doi.org/10.1080/15376490903138888.

[20] S. Benyoucef, I. Mechab, A. Tounsi, A. Fekrar, H. A. Atmane, and E. A. A. Bedia. Bending of thick functionally graded plates resting on Winkler-Pasternak elastic foundations. Mechanics of Composite Materials, 46, (4), (2010), pp. 425-434. https://doi.org/10.1007/s11029-010-9159-5.

[21] M. Sobhy. Buckling and free vibration of exponentially graded sandwich plates resting on elastic foundations under various boundary conditions. Composite Structures, 99, (2013), pp. 76-87. https://doi.org/10.1016/j.compstruct.2012.11.018.

[22] S. A. Al Khateeb and A. M. Zenkour. A refined four-unknown plate theory for advanced plates resting on elastic foundations in hygrothermal environment. Composite Structures, 111, (2014), pp. 240-248. https://doi.org/10.1016/j.compstruct.2013.12.033.

[23] H. V. Tung. Thermal and thermomechanical postbuckling of FGM sandwich plates resting on elastic foundations with tangential edge constraints and temperature dependent properties. Composite Structures, 131, (2015), pp. 1028-1039. https://doi.org/10.1016/j.compstruct.2015.06.043.

[24] N. M. Khoa and H. V. Tung. Nonlinear thermo-mechanical stability of shear deformable FGM sandwich shallow spherical shells with tangential edge constraints. Vietnam Journal of Mechanics, 39, (4), (2017), pp. 351-364. https://doi.org/10.15625/0866-7136/9810.

[25] S. S. Akavci. Mechanical behavior of functionally graded sandwich plates on elastic foundation. Composites Part B: Engineering, 96, (2016), pp. 136-152. https://doi.org/10.1016/j.compositesb.2016.04.035.

[26] R. Benferhat, T. H. Daouadji, and M. S. Mansour. Free vibration analysis of FG plates resting on an elastic foundation and based on the neutral surface concept using higherorder shear deformation theory. Comptes Rendus Mecanique, 344, (9), (2016), pp. 631-641. https://doi.org/10.12989/eas.2016.10.5.1033.

[27] A. Benahmed, M. S. A. Houari, S. Benyoucef, K. Belakhdar, and A. Tounsi. A novel quasi-3D hyperbolic shear deformation theory for functionally graded thick rectangular plates on elastic foundation. Geomechanics and Engineering, 12, (1), (2017), pp. 9-34. https://doi.org/10.12989/gae.2017.12.1.009. 
[28] F. Z. Zaoui, D. Ouinas, and A. Tounsi. New 2D and quasi-3D shear deformation theories for free vibration of functionally graded plates on elastic foundations. Composites Part B: Engineering, 159, (2019), pp. 231-247. https://doi.org/10.1016/j.compositesb.2018.09.051.

[29] M. Eisenberger, D. Z. Yankelevsky, and M. A. Adin. Vibrations of beams fully or partially supported on elastic foundations. Earthquake Engineering \& Structural Dynamics, 13, (5), (1985), pp. 651-660. https://doi.org/10.1002/eqe.4290130507.

[30] T. Yokoyama. Vibration analysis of Timoshenko beam-columns on two-parameter elastic foundations. Computers \& Structures, 61, (6), (1996), pp. 995-1007. https://doi.org/10.1016/0045-7949(96)00107-1.

[31] S. Motaghian, M. Mofid, and J. E. Akin. On the free vibration response of rectangular plates, partially supported on elastic foundation. Applied Mathematical Modelling, 36, (9), (2012), pp. 4473-4482. https://doi.org/10.1016/j.apm.2011.11.076.

[32] H. S. Shen. Functionally graded materials: nonlinear analysis of plates and shells. CRC Press, Taylor \& Francis Group, Boca Raton, (2009).

[33] R. D. Cook, D. S. Malkus, and M. E. Plesha. Concepts and applications of finite element analysis. John Willey \& Sons, New York, 3rd edition, (1989).

[34] S. S. Rao. The finite element method in engineering. Elsevier, Amsterdam, 4th edition, (2005). 